\title{
Mothers' Physical Activity in the New Millennium: A Systematic Review of the Literature
}

\author{
Ignatius Darma Juwono, Bernadette Kun, Zsolt Demetrovics, Attila Szabo \\ ELTE Eötvös Loránd University, Budapest, Hungary
}

\begin{abstract}
Background. Physical activity is essential for health, especially in an increasingly sedentary world. Being a parent involves responsibilities that could interfere with mothers' physical activity. Research suggests that mothers are especially vulnerable to physical inactivity. Health promoters invest substantial effort in facilitating the physical activity of women with children.

Methods. The scope of this systematic literature review is to synthesize research efforts on mothers' physical activity and supporting interventions. Three databases were scrutinized in this review (Medline, Scopus, and PsycINFO). Articles published between 2000-2020 were selected in concordance with the Preferred Reporting Items for Systematic Reviews and Meta-analysis (PRISMA). Fifty-four eligible articles were included after screening 3708 articles.

Results. The results suggest that mothers exhibit lower levels of physical activity than the recommended level for adults. Lack of time, focus on childcare, limited knowledge, and insufficient spousal support emerge as the primary barriers to physical activity in mothers with children. Interventions trying to overcome these barriers vary in methods, evaluations, and effectiveness. Positive changes in mothers' physical activity may emerge if interventions focus on increasing self-efficacy, problem-solving, goal setting, and other cognitive factors.

Conclusion. Future interventions integrating internet-based applications and involving spouses and/or partners might help mothers to increase their physical activity.
\end{abstract}

Keywords: exercise, health behavior, motherhood, sedentary, women.

\section{INTRODUCTION}

$\mathrm{P}$ hysical activity (PA) is a crucial determinant of overall health regardless of age (Abbasi \& van den Akker, 2015; Bellows-Riecken \& Rhodes, 2008; Hamilton \& White, 2010b; Li et al., 2012; World Health Organization [WHO], 2018). In general, adults should engage in at least 150 minutes of Moderate-to-Vigorous PA (MVPA) per week (WHO, 2011). However, recent research unveiled a significant decline in PA over the past decades (Abbasi \& van den Akker, 2015; Archer et al., 2013). The fall is more remarkable in adulthood (Jung \& Brawley, 2013) when most people need to simultaneously fulfil numerous challenges. For example, college students have to focus on academic, social, and time-management activities.
At the same time, workers have to meet their jobrelated obligations while also caring for themselves and their families (Jung \& Brawley, 2013). A major life-event related to women's fall in PA is childbearing (Hamilton \& White, 2010b).

Studies seemed to suggest that adults with dependent children tend to be physically more inactive than the general population (BellowsRiecken \& Rhodes, 2008; Gaston, Edwards, Doelman, \& Tober, 2014; Hartman, Hosper, \& Stronks, 2011). Although children's presence may affect both parents, mothers are at higher risk of being physically inactive. For example, one study estimated that mothers accrue 86 minutes less PA than fathers (Nomaguchi \& Bianchi, 2004). The 
reason for this difference, based on two research, is the mothers' role as primary childcare providers in addition to various household responsibilities (Bellows-Riecken \& Rhodes, 2008; Mcquillan, Greil, Scheffler, \& Tichenor, 2010). These roles are both time and energy consuming which could discourage mothers from engaging in regular PA levels (Abbasi \& van den Akker, 2015; BellowsRiecken \& Rhodes, 2008; Gaston et al., 2014; Hartman et al., 2011).

From the beginning of the new millennium, numerous studies have examined mothers' PA patterns, the facilitators and perceived barriers to PA, and the interventions to boost PA. However, more thorough literature reviews on this topic are limited. Two literature reviews, which included research performed in the new millennium, concluded that parenthood is inversely related to the overall PA (Abbasi \& van den Akker, 2015; Bellows-Riecken \& Rhodes, 2008). The first of the two reviews only included 24 studies examining the usually performed PA while excluding studies that used an intervention. This paper disclosed a substantial decline in the amount of moderate to strenuous PA in the transition period to motherhood, which does not generally increase again after giving birth (Abbasi \& van den Akker, 2015). The other literature review was a narrative review based on 31 articles and 25 samples that excluded adolescents' mothers; it concluded that parents with children are significantly more inactive than nonparents (Bellows-Riecken \& Rhodes, 2008). Since these reviews were published, several interventions attempted to increase mothers' PA. However, no up-to-date reviews exist on the effectiveness of these intervention programs.

This current review aims to examine mothers' PA concerning the recommended guidelines for PA for health. The WHO guideline recommends that adults engage in at least 150 minutes of moderateto-vigorous PA (MVPA) (WHO, 2011). This guideline applies to all adults, including mothers. However, the United States Center for Disease Control and Prevention (US CDC) offers a more specific recommendation for postpartum mothers. The US CDC outlines that postpartum mothers should engage in at least 150 minutes of moderate PA per week (MPA) (U.S. Department of Health and Human Services, 2018). Both guidelines are used in the current review, as the authors do not limit the child's age. Therefore, regardless of the policy, this review aims to disclose whether mothers meet the recommended level of PA. This systematic review also seeks to explore both the facilitators and barriers to mothers' regular PA. Another objective of the current analysis is to evaluate the effectiveness of interventions aimed at improving mothers' PA, which is absent from the last two published reviews. This objective is fundamental from a pragmatic perspective for the specialized training settings facilitating the PA of women with children. Apart from being more recent, the current review differs from the past two reviews in that it includes more than twice as many studies, and it also evaluates PA interventions. The authors hypothesized that women with children still do not get enough PA for health despite new interventions.

\section{METHODS}

The current systematic review employs principles outlined by the Preferred Reporting Items for Systematic Reviews and Meta-analysis (PRISMA) (Liberati et al., 2009; Moher et al., 2009). The search is delimited to articles published in the new millennium (2000-2020). In this period, there was an increased research effort to increase mothers' PA, and the last review published a few years ago (Abbasi \& van den Akker, 2015) did not include intervention studies.

Eligibility criteria. Studies inclusion criteria were:

1) Studies published in English between 20002020.

2) Original research in peer-reviewed journals.

3) Upon selection of the articles, researchers ensured that the studies meet the 'Population Intervention, Comparison, and Outcome' (PICO) standard (Liberati et al., 2009):

a. $\mathrm{P}$ (population): mothers (postpartum women, mothers of toddlers, schoolaged children, and teenagers) of any socioeconomic and marital status, free of health condition preventing them from engaging in regular PA.

b. I (intervention): is not necessary. Studies that measured PA level in mothers using an objective accelerometer or subjective self-reports are included. Any intervention studies included should report the impact of the intervention on mothers' PA.

c. C (comparison): mothers' PA level are compared with adult's recommended level of PA outlined by the WHO (150 minutes of 
MVPA per week) or US CDC (150 minutes of MPA per week). For intervention studies, the use of a control group is required to ensure the differences between groups that could be ascribed to the treatment effects.

d. O (Output): the studies need to identify the levels of PA in mothers. In correlational and cross-sectional studies, variables directly associated with mothers' PA should be examined/reported. Finally, for intervention studies, changes or differences in PA levels between the treatment and control groups should be reported.

Studies with parents (father-mother) or parentchild dyads are included if they provide information on the mothers' PA. Studies comparing PA prior the pregnancy and postpartum are also included. However, studies focusing on pregnant women's PA are excluded since the focus of this review is not on how pregnancy impacts women's PA. Reviews articles, dissertations, non-English publications, and technical papers are excluded from this review.

Search strategy. Three electronic databases (Scopus, PubMed/Medline, and PsycINFO) were searched. A combination of keywords and filters were utilized. The Boolean logic-commands were used during the search. The results were sorted by relevance. Other than searching through the databases, the authors also looked at the references in the relevant articles. The search strategy is outlined in Table 1.

The search from three databases yielded 3685 articles. Examination of the references in the 3685 articles and search through Google Scholar provided 23 more articles. After identifying duplicates, 40 articles were excluded. The examination of the titles and abstracts resulted in the exclusion of 3466 articles. From the remaining 202 eligible articles, 87 were excluded for various non-eligibility or non-accessibility reasons leaving 115 full text articles to be read. These were screening for Population, Information, Comparison, and Outcomes (PICO). Articles not focusing on mothers' PA or relating it to adults' or postpartum PA guidelines $(n=61)$ were then removed. The final number of articles included in the review was 54, which focused on PA of mothers (level of engagement; barriers or facilitators to PA; and intervention to increase PA level in mothers). The selection process is summarized in Figure 2, and a summary of included articles is listed in Table 3.

Data extraction and analysis. From the selected studies, data regarding authors (and year of publication); study design and methods, characteristics of the sample; PA measurements used; and primary outcome of the study were extracted. For studies focusing on intervention effectiveness, additional information on both the nature and duration of the intervention was also extracted. One author extracted the relevant information from the selected articles (IDJ).

Quality assessment and risk of bias. In this systematic review, we adopted the risk of bias in systematic review (ROBIS) tool. The ROBIS assesses the risk of bias in three phases: Phase 1: assessing the relevance, which is an optional phase; Phase 2: assessing the review process; and Phase 3: assessing bias in the review itself. It was recommended that ROBIS should assess at least Phase 2 and 3. Phase 2 is comprised of 4 domains: study eligibility criteria; identification and selection of studies; data collection and study appraisal; and synthesis and findings. Each domain has 5 signalling questions, except for the synthesis and findings (which has 6 signalling questions). Phase 3 is guided by three signalling questions. Despite its novelty, the ROBIS is robust and has good construct validity in assessing the risk of bias.

\begin{tabular}{l|l|l|}
\cline { 2 - 3 } $\begin{array}{l}\text { Table 1. Details of the } \\
\text { search strategies used } \\
\text { in current review }\end{array}$ & Search Strategy Items & \\
\cline { 2 - 3 } & Used keywords & (mothers OR “women with children”) AND (physical activity OR “exercise”) \\
\hline & Searched databases & PubMed/MEDLINE, Scopus, and PsycINFO \\
\hline Time filter & $2000-2020$ \\
\hline Language filter & English only \\
\cline { 2 - 3 } & Document type filter & Articles in peer-reviewed journals \\
\hline Inclusion criteria & Original article complying to the established PICO \\
\cline { 2 - 3 } & Exclusion criteria & Reviews, Dissertation, Technical papers \\
\hline
\end{tabular}


Ignatius Darma Juwono, Bernadette Kun, Zsolt Demetrovics, Attila Szabo MOTHERS' PHYSICAL ACTIVITY IN THE NEW MILLENNIUM: A SYSTEMATIC REVIEW OF THE LITERATURE

Table 2. ROBIS risk assessment of bias in the systematic review summary
Notes. '+'- high risk; '-‘- low risk; ID - identification; In the ROBIS system each domain is guided by few signalling questions. If all the signaling questions are answered "Yes" or "Probably Yes", the concern of bias is low. But, if any signalling question is answered "no" or "probably no", potential risk of bias is high (Whiting et al., 2016). The use of filter especially in non-RCT study would increase the likelihood of some eligible studies excluded, heightening the risk of bias (Whiting et al., 2016).

\begin{tabular}{|c|c|c|c|c|c|c|}
\hline \multirow[b]{2}{*}{ 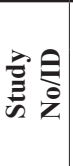 } & \multirow[b]{2}{*}{$\begin{array}{l}\text { Author(s) } \\
\text { Year }\end{array}$} & \multicolumn{4}{|c|}{ Phase 2} & \multirow{2}{*}{$\begin{array}{c}\text { Phase } 3 \\
\text { Risk of } \\
\text { bias in the } \\
\text { review }\end{array}$} \\
\hline & & $\begin{array}{l}\text { 1. Eligi- } \\
\text { biity } \\
\text { criteria }\end{array}$ & $\begin{array}{c}\text { 2. ID \& } \\
\text { selection of } \\
\text { studies }\end{array}$ & $\begin{array}{l}\text { 3. Data collec- } \\
\text { tion and study } \\
\text { appraisal }\end{array}$ & $\begin{array}{l}\text { 4. Synt- } \\
\text { hesis \& } \\
\text { findings }\end{array}$ & \\
\hline 1 & Brown et al. (2001) & - & + & - & - & - \\
\hline 2 & Sallis et al. (2001) & - & + & + & - & + \\
\hline 3 & Miller et al. (2002) & - & + & - & - & - \\
\hline 4 & Ransdell et al. (2003) & - & + & - & - & - \\
\hline 5 & Albright et al. (2005) & - & - & + & + & + \\
\hline 6 & Bell \& Lee (2005) & - & + & - & - & - \\
\hline 7 & Clarke et al. (2007) & - & + & - & - & - \\
\hline 8 & Cleland et al. (2008) & - & + & - & - & - \\
\hline 9 & Albright et al. (2009) & - & + & + & + & + \\
\hline 10 & Evenson et al. (2009) & - & + & - & - & - \\
\hline 11 & Lombard et al. (2009) & - & - & - & - & - \\
\hline 12 & Adachi-Mejia et al. (2010) & - & + & + & - & + \\
\hline 13 & Cramp \& Bray (2010) & - & + & - & - & - \\
\hline 14 & Hamilton \& White (2010b) & - & + & - & + & + \\
\hline 15 & Hamilton \& White (2010a) & - & + & - & - & - \\
\hline 16 & Hull et al. (2010) & - & - & - & - & - \\
\hline 17 & Norman et al. (2010) & - & - & - & - & - \\
\hline 18 & Dombrowski (2011) & - & + & - & - & - \\
\hline 19 & Hamilton \& White (2011) & - & + & - & - & - \\
\hline 20 & Jung \& Brawley (2011) & - & + & - & - & - \\
\hline 21 & Olvera et al. (2011) & - & + & - & - & - \\
\hline 22 & Schluter et al. (2011) & - & + & - & - & - \\
\hline 23 & Albright et al. (2011) & - & - & - & - & - \\
\hline 24 & Candelaria et al. (2012) & - & + & - & - & - \\
\hline 25 & Dearth-Wesley et al. (2012) & - & + & + & - & + \\
\hline 26 & Mansfield et al. (2012) & - & + & + & - & + \\
\hline 27 & Dlugonski \& Motl (2013) & - & + & - & - & - \\
\hline 28 & McGannon \& Schinke (2013) & - & + & + & + & + \\
\hline 29 & Monteiro et al. (2013) & - & - & - & - & - \\
\hline 30 & Hesketh et al. (2014) & - & + & - & - & - \\
\hline 31 & Keller et al. (2014) & - & - & - & - & - \\
\hline 32 & Mailey et al. (2014) & - & + & - & + & + \\
\hline 33 & Mailey \& McAuley (2014) & - & - & - & - & - \\
\hline 34 & Rhodes et al. (2014) & - & + & - & - & - \\
\hline 35 & Fjeldsoe et al. (2015) & - & - & - & - & - \\
\hline 36 & Jiryaee et al. (2015) & - & - & - & - & - \\
\hline 37 & Lovell \& Butler (2015) & - & + & + & - & - \\
\hline 38 & Bronikowksi et al. (2016) & - & + & - & + & + \\
\hline 39 & Cotter et al. (2016) & - & + & - & + & + \\
\hline 40 & Dlugonski \& Motl (2016) & - & + & - & + & + \\
\hline 41 & Gierc et al. (2016) & - & + & - & - & - \\
\hline 42 & Lloyd et al. (2016) & - & + & - & + & + \\
\hline 43 & Mailey et al. (2016) & - & + & - & - & - \\
\hline 44 & Saligheh et al. (2016) & - & + & - & - & - \\
\hline 45 & Tuominen et al. (2016) & - & - & - & - & - \\
\hline 46 & Dlugonski et al. (2017a) & - & + & - & - & - \\
\hline 47 & Dlugonski et al. (2017b) & - & + & - & - & - \\
\hline 48 & Khodabandeh et al. (2017) & - & - & - & - & - \\
\hline 49 & Mailey \& Hsu (2017) & - & - & - & - & - \\
\hline 50 & Suzuki et al. (2017) & - & + & - & - & - \\
\hline 51 & Alhassan et al (2018) & - & - & - & - & - \\
\hline 52 & Carson et al. (2018) & - & + & - & - & - \\
\hline 53 & Mascarenhas et al. (2018) & - & - & - & - & - \\
\hline 54 & Carver et al. (2020) & - & + & - & - & - \\
\hline
\end{tabular}


Table 3. Summary of studies included in the current systematic literature review

\begin{tabular}{|c|c|c|c|c|c|c|c|c|c|c|c|c|c|c|}
\hline \multirow{2}{*}{ 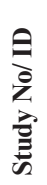 } & \multirow[b]{2}{*}{ 产 } & \multirow[b]{2}{*}{ 己̇ } & \multirow[b]{2}{*}{ Z } & \multicolumn{3}{|c|}{ Sample Characteristics } & \multirow[b]{2}{*}{ 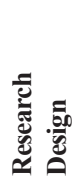 } & \multirow[b]{2}{*}{ 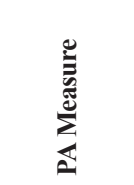 } & \multirow{2}{*}{ 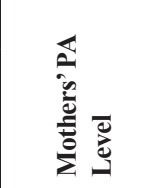 } & \multirow[b]{2}{*}{ 离 } & \multirow[b]{2}{*}{ 宸 } & \multicolumn{3}{|c|}{ Intervention } \\
\hline & & & & 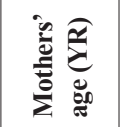 & 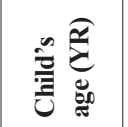 & 을 & & & & & & है & 气ี & 吾 \\
\hline 1 & $\begin{array}{l}\text { Brown et } \\
\text { al. }(2001)\end{array}$ & Australia & 543 & $33( \pm 4.8)$ & - & $\begin{array}{c}\text { FT, PT, } \\
\text { housewife }\end{array}$ & $\mathrm{CS}$ & Self-report & Insufficient PA & $\begin{array}{l}\text { Child care, } \\
\text { lack of time, } \\
\text { lack of time }\end{array}$ & - & - & - & - \\
\hline 2 & $\begin{array}{l}\text { Sallis et al. } \\
(2001)\end{array}$ & US & 226 & $\geq 18$ & \pm 4 & - & LG & Self-report & $\begin{array}{l}\text { Increase } \\
\text { in second } \\
\text { measure }\end{array}$ & - & - & - & - & - \\
\hline 3 & $\begin{array}{l}\text { Miller et al. } \\
(2002)\end{array}$ & Australia & 554 & $\geq 18$ & - & - & $\mathrm{RCT}$ & Self-report & $\begin{array}{c}\text { Insufficient PA } \\
\text { at baseline }\end{array}$ & & $\begin{array}{l}\text { Social sup- } \\
\text { port }\end{array}$ & $\begin{array}{c}\text { Printed } \\
\text { education \& } \\
\text { goal setting } \\
\text { strategies }\end{array}$ & $\begin{array}{c}8 \\
\text { weeks }\end{array}$ & Sig. \\
\hline 4 & $\begin{array}{l}\text { Ransdell et } \\
\text { al. (2003) }\end{array}$ & US & 34 & $31-60$ & $14-16$ & - & RCT & Self-report & - & - & - & $\begin{array}{l}\text { Home-based } \\
\text { vs communi- } \\
\text { ty-based PA }\end{array}$ & $\begin{array}{c}12 \\
\text { weeks }\end{array}$ & Sig. \\
\hline 5 & $\begin{array}{l}\text { Albright et } \\
\text { al. (2005) }\end{array}$ & US & 79 & $\geq 18$ & $\begin{array}{l}\text { 2-18 } \\
\text { months }\end{array}$ & - & MM & Self-report & $\begin{array}{c}\text { Significant } \\
\text { proportion } \\
\text { were active } \\
\text { before preg- } \\
\text { nancy but } \\
\text { were inactive } \\
\text { now }\end{array}$ & $\begin{array}{l}\text { Personal } \\
\text { issue and } \\
\text { parenting } \\
\text { duties }\end{array}$ & $\begin{array}{c}\text { Social } \\
\text { support, } \\
\text { help for } \\
\text { childcare, } \\
\text { perceived } \\
\text { benefits }\end{array}$ & - & - & - \\
\hline 6 & $\begin{array}{l}\text { Bell \& Lee } \\
(2005)\end{array}$ & Australia & 8545 & $18-23$ & - & - & LG & Self-report & $\begin{array}{c}\text { Larger decline } \\
\text { in PA in moth- } \\
\text { ers than non- } \\
\text { mothers }\end{array}$ & - & - & - & - & - \\
\hline 7 & $\begin{array}{l}\text { Clarke et } \\
\text { al. }(2007)\end{array}$ & US & 124 & $18-45$ & $1-4$ & - & RCT & Objective & - & - & - & $\begin{array}{c}\text { PA, health } \\
\text { eating week- } \\
\text { ly lessons }\end{array}$ & $\begin{array}{c}8 \\
\text { weeks }\end{array}$ & Sig. \\
\hline 8 & $\begin{array}{l}\text { Cleland et } \\
\text { al. (2008) }\end{array}$ & Australia & 357 & $\begin{array}{c}42.4 \\
( \pm 5.1)\end{array}$ & $\leq 12$ & - & CR & Self-report & - & - & $\begin{array}{c}\text { Social sup- } \\
\text { port, quality } \\
\text { of facilities, } \\
\text { safety }\end{array}$ & - & - & - \\
\hline 9 & $\begin{array}{l}\text { Albright et } \\
\text { al. (2009) }\end{array}$ & US & 20 & $33( \pm 3.8)$ & $\begin{array}{c}6.9( \pm 2.4) \\
\text { months }\end{array}$ & - & WS & Self-report & $\begin{array}{c}\text { Insufficient PA } \\
\text { at baseline }\end{array}$ & - & - & $\begin{array}{l}\text { SCT based } \\
\text { intervention }\end{array}$ & $\mid \begin{array}{c}2 \\
\text { months }\end{array}$ & Sig. \\
\hline 10 & $\begin{array}{l}\text { Evenson et } \\
\text { al. (2009) }\end{array}$ & US & 667 & $20-34$ & $\begin{array}{c}\leq 6 \\
\text { months }\end{array}$ & - & LG & Self-report & $\begin{array}{c}\text { Insufficient } \\
\text { MVPA at } 3 \\
\text { months post- } \\
\text { partum }\end{array}$ & $\begin{array}{c}\text { Lack of time, } \\
\text { child care }\end{array}$ & $\begin{array}{c}\text { Partner } \\
\text { support, } \\
\text { motivation } \\
\text { feel-good }\end{array}$ & - & - & - \\
\hline 11 & $\begin{array}{l}\text { Lombard et } \\
\text { al. (2009) }\end{array}$ & Australia & 254 & $\geq 18$ & $5-13$ & - & RCT & Self-report & - & - & - & $\begin{array}{c}\text { Self-man- } \\
\text { agement } \\
\text { program }\end{array}$ & $\begin{array}{c}4 \\
\text { months }\end{array}$ & NS \\
\hline 12 & $\begin{array}{l}\text { Adachi- } \\
\text { Mejia et al. } \\
(2010)\end{array}$ & US & 1691 & $40-49$ & $9-12$ & - & $\mathrm{CR}$ & Self-report & $\begin{array}{c}\text { Reported } \\
\text { sufficient PA } \\
\text { level per week }\end{array}$ & $\begin{array}{l}\text { Lack of } \\
\text { time, lack } \\
\text { of energy } \\
\text { and self- } \\
\text { discipline }\end{array}$ & - & - & - & - \\
\hline 13 & $\begin{array}{l}\text { Cramp } \\
\text { \& Bray } \\
(2010)\end{array}$ & US & 23 & $\begin{array}{c}29 \\
( \pm 3.48)\end{array}$ & $\begin{array}{c}2-10 \\
\text { months }\end{array}$ & - & $\mathrm{CSO}$ & Self-report & - & - & - & $\begin{array}{l}\text { Exercise } \\
\text { with or- } \\
\text { 4without } \\
\text { baby }\end{array}$ & $\begin{array}{c}2 \\
\text { weeks }\end{array}$ & NS \\
\hline 14 & $\begin{array}{l}\text { Hamilton } \\
\text { \& White } \\
\text { (2010a) }\end{array}$ & Australia & 21 & $\geq 18$ & $\leq 5$ & - & Qual. & Self-report & $\begin{array}{c}\text { Reported } \\
\text { change in PA } \\
\text { since having } \\
\text { child }\end{array}$ & - & $\begin{array}{c}\text { Desire to } \\
\text { live longer } \\
\text { to see child } \\
\text { grows }\end{array}$ & - & - & - \\
\hline
\end{tabular}


Table 3. (continued)

\begin{tabular}{|c|c|c|c|c|c|c|c|c|c|c|c|c|c|c|}
\hline \multirow{2}{*}{ 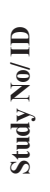 } & \multirow[b]{2}{*}{ 产 } & \multirow[b]{2}{*}{ 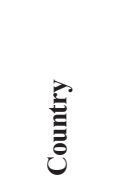 } & \multirow[b]{2}{*}{ Z } & \multicolumn{3}{|c|}{ Sample Characteristics } & \multirow[b]{2}{*}{ 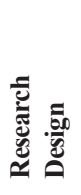 } & \multirow[b]{2}{*}{ 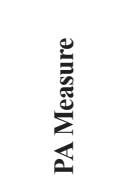 } & \multirow{2}{*}{ 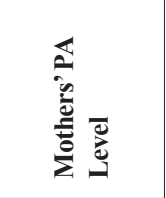 } & \multirow[b]{2}{*}{ คับ } & \multirow[b]{2}{*}{ : } & \multicolumn{3}{|c|}{ Intervention } \\
\hline & & & & 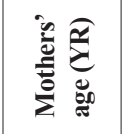 & $\sum_{\substack{0 \\
0}}^{\infty}$ & 을 & & & & & & $\sum^{0}$ & 氙 & 节 \\
\hline 15 & $\begin{array}{l}\text { Hamilton } \\
\text { \& White } \\
(2010 c)\end{array}$ & Australia & 21 & $\geq 18$ & - & - & Qual. & Self-report & - & $\begin{array}{c}\text { Beliefs of PA } \\
\text { will interfere } \\
\text { other roles }\end{array}$ & - & - & - & - \\
\hline 16 & $\begin{array}{l}\text { Hull et al. } \\
(2010)\end{array}$ & US & 349 & $\geq 18$ & - & $\begin{array}{c}\text { FT, PT } \\
\text { and other }\end{array}$ & LG & Self-report & $\begin{array}{c}\text { Reported PA } \\
\text { decrease since } \\
\text { having child }\end{array}$ & - & - & - & - & - \\
\hline 17 & $\begin{array}{l}\text { Norman et } \\
\text { al. (2010) }\end{array}$ & Australia & 135 & $17-40$ & $\begin{array}{c}\text { 6-10 } \\
\text { months }\end{array}$ & - & $\mathrm{RCT}$ & Self-report & - & - & - & $\begin{array}{l}\text { Mother } \\
+ \text { baby } \\
\text { exercise }\end{array}$ & $\begin{array}{c}8 \\
\text { weeks }\end{array}$ & NS \\
\hline 18 & $\begin{array}{l}\text { Dombrowski } \\
(2011)\end{array}$ & US & 110 & $34-44$ & $\leq 18$ & FT & $\mathrm{CR}$ & Self-report & Insufficient & - & $\begin{array}{l}\text { Self- } \\
\text { efficacy }\end{array}$ & - & - & - \\
\hline 19 & $\begin{array}{l}\text { Hamilton } \\
\text { \& White } \\
\text { (2011) }\end{array}$ & Australia & 288 & $\geq 18$ & - & - & $\mathrm{CS}$ & Self-report & - & - & $\begin{array}{c}\text { Perceived } \\
\text { benefit of } \\
\text { PA and } \\
\text { spousal } \\
\text { support }\end{array}$ & - & - & - \\
\hline 20 & $\begin{array}{l}\text { Jung \& } \\
\text { Brawley } \\
(2011)\end{array}$ & Canada & 49 & $18-49$ & $\leq 10$ & FT & $\mathrm{R}$ & Self-report & - & - & $\begin{array}{c}\text { Self- } \\
\text { regulation } \\
\text { efficacy }\end{array}$ & - & - & - \\
\hline 21 & $\begin{array}{l}\text { Olvera et } \\
\text { al. }(2011)\end{array}$ & US & 102 & $\begin{array}{c}36.3 \\
( \pm 7.2)\end{array}$ & $\leq 10$ & - & $\mathrm{CS}$ & Objective & Insufficient PA & - & - & - & - & - \\
\hline 22 & $\begin{array}{l}\text { Schluter et } \\
\text { al. }(2011)\end{array}$ & $\begin{array}{c}\text { New } \\
\text { Zealand }\end{array}$ & 2244 & $20-54$ & $5-15$ & - & MM & Objective & Insufficient PA & $\begin{array}{c}\text { Lack of } \\
\text { time, energy, } \\
\text { difficulty } \\
\text { to make PA } \\
\text { routine }\end{array}$ & $\begin{array}{l}\text { Low PA, } \\
\text { facilities' } \\
\text { fee, extra } \\
\text { time, role } \\
\text { model for } \\
\text { child }\end{array}$ & - & - & - \\
\hline 23 & $\begin{array}{l}\text { Albright et } \\
\text { al. (2012) }\end{array}$ & US & 278 & $32( \pm 5.6)$ & $\begin{array}{c}\leq 12 \\
\text { months }\end{array}$ & - & $\mathrm{RCT}$ & $\begin{array}{l}\text { Objective } \\
\text { and Self- } \\
\text { report }\end{array}$ & $\begin{array}{c}\text { Insufficient at } \\
\text { baseline }\end{array}$ & $\begin{array}{c}\text { Social } \\
\text { support from } \\
\text { partner }\end{array}$ & - & - & - & - \\
\hline 24 & $\begin{array}{l}\text { Candelaria } \\
\text { et al. } \\
(2012)\end{array}$ & US & 909 & $20-58$ & - & - & $\mathrm{CS}$ & $\begin{array}{l}\text { Objective } \\
\text { and Self- } \\
\text { report }\end{array}$ & $\begin{array}{c}\text { No difference } \\
\text { in objectively } \\
\text { measured } \\
\text { PA between } \\
\text { mothers and } \\
\text { non-mothers }\end{array}$ & - & - & - & - & - \\
\hline 25 & $\begin{array}{l}\text { Dearth- } \\
\text { Wesley et } \\
\text { al. (2012) }\end{array}$ & China & 353 & $\geq 18$ & $6-9$ & - & LG & Self-report & $\begin{array}{c}\text { Increase in PA } \\
\text { but decline } \\
\text { in active } \\
\text { commuting }\end{array}$ & - & - & - & - & - \\
\hline 26 & $\begin{array}{l}\text { Mansfield } \\
\text { et al. } \\
(2012)\end{array}$ & Canada & 42 & $35( \pm 7.7)$ & $\leq 14$ & - & MM & Self-report & $\begin{array}{c}\text { No differences } \\
\text { of PA among } \\
\text { employed and } \\
\text { unemployed }\end{array}$ & $\begin{array}{c}\text { Time } \\
\text { constraint, } \\
\text { childcare, } \\
\text { lack } \\
\text { motivation, } \\
\text { lack of } \\
\text { knowledge }\end{array}$ & $\begin{array}{c}\text { Access to } \\
\text { PA facilities } \\
\text { and safety }\end{array}$ & - & - & - \\
\hline 27 & $\begin{array}{l}\text { Dlugonski } \\
\text { \& Motl } \\
(2013)\end{array}$ & US & 66 & $18-64$ & $\leq 18$ & - & $\mathrm{CS}$ & $\begin{array}{l}\text { Objective } \\
\text { and Self- } \\
\text { report }\end{array}$ & $\begin{array}{l}\text { PA level } \\
\text { differences } \\
\text { among } \\
\text { mothers and } \\
\text { nonmothers }\end{array}$ & - & - & - & - & - \\
\hline
\end{tabular}


Table 3. (continued)

\begin{tabular}{|c|c|c|c|c|c|c|c|c|c|c|c|c|c|c|}
\hline \multirow{2}{*}{ 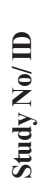 } & \multirow[b]{2}{*}{ 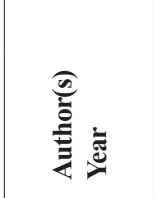 } & \multirow[b]{2}{*}{$\stackrel{\Xi}{E}$} & \multirow[b]{2}{*}{ Z } & \multicolumn{3}{|c|}{ Sample Characteristics } & \multirow[b]{2}{*}{ 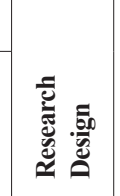 } & \multirow[b]{2}{*}{ 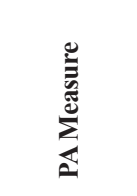 } & \multirow[b]{2}{*}{ 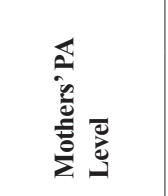 } & \multirow[b]{2}{*}{ 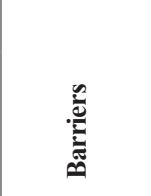 } & \multirow[b]{2}{*}{ 気 } & \multicolumn{3}{|c|}{ Intervention } \\
\hline & & & & 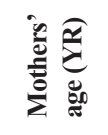 & 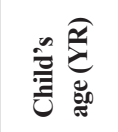 & 을 & & & & & & $\sum_{1}^{\infty}$ & 気 & 节 \\
\hline 28 & $\begin{array}{l}\text { McGannon } \\
\text { \& Schinke } \\
(2013)\end{array}$ & Canada & 1 & 35 & $\leq 4$ & FT & $\begin{array}{l}\text { Case } \\
\text { study }\end{array}$ & Self-report & - & $\begin{array}{c}\text { Guilt, family } \\
\text { care }\end{array}$ & \begin{tabular}{|} 
Perceived \\
benefit of \\
PA and \\
spousal \\
support
\end{tabular} & - & - & - \\
\hline 29 & $\begin{array}{l}\text { Monteiro et } \\
\text { al. (2013) }\end{array}$ & Australia & 716 & $\geq 25$ & $1-4$ & - & $\mathrm{RCT}$ & $\begin{array}{l}\text { Objective } \\
\text { and Self- } \\
\text { report }\end{array}$ & - & - & - & $\begin{array}{c}\text { Home- } \\
\text { based PA } \\
\text { intervention }\end{array}$ & $\begin{array}{c}6 \\
\text { months }\end{array}$ & Sig. \\
\hline 30 & $\begin{array}{l}\text { Hesketh et } \\
\text { al. (2014) }\end{array}$ & UK & 554 & $20-34$ & $\begin{array}{l}\text { Pre-- } \\
\text { school }\end{array}$ & - & $\mid \begin{array}{c}\text { Popula- } \\
\text { tion study }\end{array}$ & Objective & Insufficient & - & - & - & - & - \\
\hline 31 & $\begin{array}{l}\text { Keller et al. } \\
(2014)\end{array}$ & US & 139 & $18-40$ & $<12$ & - & $\mathrm{RCT}$ & $\begin{array}{l}\text { Objective } \\
\text { and Self- } \\
\text { report }\end{array}$ & - & - & - & \begin{tabular}{|c|} 
Social \\
Support \\
Intervention
\end{tabular} & $\begin{array}{c}12 \\
\text { weeks }\end{array}$ & Sig. \\
\hline 32 & $\begin{array}{l}\text { Mailey et al. } \\
(2014)\end{array}$ & US & 13 & $18-50$ & $\leq 18$ & FT & Qual. & Self-report & - & \begin{tabular}{|} 
Family care, \\
childcare, \\
guilt, lack \\
of support, \\
scheduling \\
constraint
\end{tabular} & $\begin{array}{c}\text { Role model } \\
\text { for children, } \\
\text { having } \\
\text { support, } \\
\text { perceived } \\
\text { benefits }\end{array}$ & - & - & - \\
\hline 33 & $\begin{array}{l}\text { Mailey \& } \\
\text { McAuley } \\
(2014)\end{array}$ & US & 141 & $25-52$ & $\leq 12$ & FT & RCT & $\begin{array}{l}\text { Objective } \\
\text { and Self- } \\
\text { report }\end{array}$ & - & - & - & Workshop & $\begin{array}{c}1 \\
\text { month }\end{array}$ & Sig. \\
\hline 34 & $\begin{array}{l}\text { Rhodes et al. } \\
(2014)\end{array}$ & Canada & 238 & $25-40$ & & - & LG & Objective & $\begin{array}{c}\text { First time } \\
\text { mothers had } \\
\text { larger decrease } \\
\text { in PA }\end{array}$ & - & $\begin{array}{c}\text { Beliefs of } \\
\text { perceived } \\
\text { benefits }\end{array}$ & - & - & - \\
\hline 35 & $\begin{array}{l}\text { Fjeldsoe et } \\
\text { al. (2015) }\end{array}$ & Australia & 263 & $\begin{array}{c}32.4 \\
( \pm 5.5)\end{array}$ & $\begin{array}{c}6 \text { weeks- } \\
5 \text { YR }\end{array}$ & - & RCT & $\begin{array}{l}\text { Objective } \\
\text { and Self- } \\
\text { report }\end{array}$ & - & - & - & $\begin{array}{l}\text { Mobile- } \\
\text { Mums } \\
\text { application }\end{array}$ & $\begin{array}{c}13 \\
\text { weeks }\end{array}$ & Sig. \\
\hline 36 & $\begin{array}{l}\text { Jiryaee et al. } \\
(2015)\end{array}$ & Iran & 172 & $\geq 18$ & $1-5$ & - & $\mathrm{RCT}$ & Self-report & $\begin{array}{c}\text { Insufficient at } \\
\text { baseline }\end{array}$ & - & - & $\begin{array}{c}\text { Goal setting } \\
\text { strategy }\end{array}$ & $\begin{array}{c}4 \\
\text { weeks }\end{array}$ & Sig. \\
\hline 37 & $\begin{array}{l}\text { Lovell } \\
\& \text { Butler } \\
(2015)\end{array}$ & Australia & 331 & $\geq 18$ & $\leq 14$ & - & $\mathrm{CS}$ & Self-report & Insufficient & - & - & - & - & - \\
\hline 38 & $\begin{array}{l}\text { Bronikowksi } \\
\text { et al. (2016) }\end{array}$ & Poland & 22 & $\begin{array}{c}38.86 \\
( \pm 2.96)\end{array}$ & $\begin{array}{c}7.96 \\
( \pm 0.69)\end{array}$ & - & WS & Self-report & - & - & - & $\begin{array}{l}\text { Parents child } \\
\text { joint exercise }\end{array}$ & $\begin{array}{c}15 \\
\text { weeks }\end{array}$ & NS \\
\hline 39 & $\begin{array}{l}\text { Cotter et al. } \\
(2016)\end{array}$ & US & 11 & $\geq 18$ & - & - & Qual. & Self-report & - & \begin{tabular}{|} 
Lack of \\
knowledge, \\
social \\
support, \\
safety, access \\
and financial
\end{tabular} & - & - & - & - \\
\hline 40 & $\begin{array}{l}\text { Dlugonski \& } \\
\text { Motl (2016) }\end{array}$ & US & 14 & $18-64$ & $\leq 18$ & - & Qual. & Self-report & - & $\begin{array}{l}\text { Lack of time, } \\
\text { child care }\end{array}$ & $\begin{array}{c}\text { Social } \\
\text { support }\end{array}$ & - & - & - \\
\hline 41 & $\begin{array}{l}\text { Gierc et al. } \\
(2016)\end{array}$ & Canada & 74 & $18-49$ & $\leq 10$ & FT & $\mathrm{MM}$ & Self-report & Insufficient & $\begin{array}{c}\text { Concurrent } \\
\text { Self- } \\
\text { Regulation } \\
\text { Efficacy }\end{array}$ & - & - & - & - \\
\hline
\end{tabular}


Table 3. (continued)

\begin{tabular}{|c|c|c|c|c|c|c|c|c|c|c|c|c|c|c|}
\hline \multirow{2}{*}{ 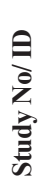 } & \multirow[b]{2}{*}{ 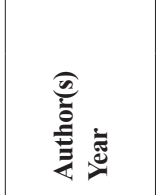 } & \multirow[b]{2}{*}{ 己̇ } & \multirow[b]{2}{*}{ Z } & \multicolumn{3}{|c|}{ Sample Characteristics } & \multirow[b]{2}{*}{ 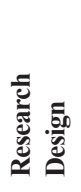 } & \multirow[b]{2}{*}{ 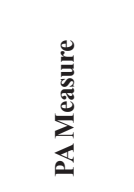 } & \multirow[b]{2}{*}{ 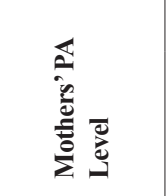 } & \multirow[b]{2}{*}{ 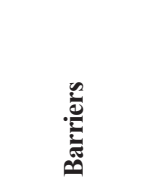 } & \multirow[b]{2}{*}{ 帝 } & \multicolumn{3}{|c|}{ Intervention } \\
\hline & & & & 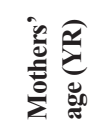 & $\sum_{\substack{0 \\
己}}^{\infty}$ & 을 & & & & & & $\sum_{i}^{2}$ & 気 & $\stackrel{\Xi}{\mathscr{Z}}$ \\
\hline 42 & $\begin{array}{l}\text { Lloyd et al. } \\
\text { (2016) }\end{array}$ & Australia & 18 & $26-41$ & - & - & Qual. & Self-report & - & Guilt & $\begin{array}{c}\text { Outcome } \\
\text { expectations, } \\
\text { intention } \\
\text { to be role } \\
\text { model }\end{array}$ & - & - & - \\
\hline 43 & $\begin{array}{l}\text { Mailey et al. } \\
(2016)\end{array}$ & US & 69 & $\begin{array}{c}35.88 \\
( \pm 5.14)\end{array}$ & $\leq 12$ & FT & $\mathrm{R}$ & $\begin{array}{l}\text { Objective } \\
\text { and Self- } \\
\text { report }\end{array}$ & - & - & - & $\begin{array}{c}\text { Online } \\
\text { intervention } \\
\text { and group } \\
\text { dynamic } \\
\text { session }\end{array}$ & $\begin{array}{c}8 \\
\text { weeks }\end{array}$ & Sig. \\
\hline 44 & $\begin{array}{l}\text { Saligheh et } \\
\text { al. (2016) }\end{array}$ & Australia & 14 & $18-43$ & $\begin{array}{c}\leq 12 \\
\text { months }\end{array}$ & - & Qual. & Self-report & & $\begin{array}{c}\text { Child and } \\
\text { family care, } \\
\text { access to PA }\end{array}$ & $\begin{array}{l}\text { Social } \\
\text { support, } \\
\text { social } \\
\text { network }\end{array}$ & - & - & - \\
\hline 45 & $\begin{array}{l}\text { Tuominen et } \\
\text { al. (2016) }\end{array}$ & Finland & 24 & $\geq 18$ & $4-7$ & - & BS & $\begin{array}{c}\text { Objective } \\
\text { and Self- } \\
\text { report }\end{array}$ & - & - & - & $\begin{array}{l}\text { Video music } \\
\text { for mother- } \\
\text { daughter }\end{array}$ & $\begin{array}{c}2 \\
\text { weeks }\end{array}$ & NS \\
\hline 46 & $\begin{array}{l}\text { Dlugonski et } \\
\text { al. }(2017 \mathrm{a})\end{array}$ & US & 86 & $18-64$ & $\leq 26$ & - & $\mathrm{CS}$ & Objective & - & $\begin{array}{c}\text { Social } \\
\text { support, } \\
\text { social } \\
\text { cohesion }\end{array}$ & - & - & - & - \\
\hline 47 & $\begin{array}{l}\text { Dlugonski et } \\
\text { al. }(2017 b)\end{array}$ & US & 32 & $18-53$ & - & $\begin{array}{c}\text { FT, PT } \\
\text { and other }\end{array}$ & Qual. & Self-report & $\begin{array}{c}\text { Insufficient PA } \\
\text { level }\end{array}$ & $\begin{array}{l}\text { Financial } \\
\text { constraint }\end{array}$ & $\begin{array}{c}\text { Role model, } \\
\text { perceived } \\
\text { benefits }\end{array}$ & - & - & - \\
\hline 48 & $\begin{array}{l}\text { Khodabandeh } \\
\text { et al. (2017) }\end{array}$ & Iran & 220 & $\geq 18$ & - & - & RCT & Self-report & - & - & - & $\begin{array}{l}\text { Lifestyle } \\
\text { education }\end{array}$ & $\begin{array}{c}12 \\
\text { weeks }\end{array}$ & NS \\
\hline 49 & $\begin{array}{l}\text { Mailey \& } \\
\text { Hsu (2017) }\end{array}$ & US & 49 & $25-40$ & $\begin{array}{c}\leq 12 \\
\text { months }\end{array}$ & - & $\mathrm{RCT}$ & $\begin{array}{l}\text { Objective } \\
\text { and Self- } \\
\text { report }\end{array}$ & & & & $\begin{array}{l}\text { Type of } \\
\text { recommen- } \\
\text { dation }\end{array}$ & $\begin{array}{c}8 \\
\text { weeks }\end{array}$ & Sig. \\
\hline 50 & $\begin{array}{l}\text { Suzuki et al. } \\
(2017)\end{array}$ & Japan & 182 & $\geq 18$ & $3-5$ & $\begin{array}{c}\text { FT, PT } \\
\text { and other }\end{array}$ & $\mathrm{CS}$ & Self-report & $\begin{array}{c}\text { Insufficient PA } \\
\text { level }\end{array}$ & & & & & \\
\hline 51 & $\begin{array}{l}\text { Alhassan et } \\
\text { al. (2018) }\end{array}$ & US & 76 & $\begin{array}{c}37.4 \\
( \pm 7.7)\end{array}$ & $7-10$ & - & $\mathrm{RCT}$ & Objective & $\begin{array}{c}\text { Insufficient at } \\
\text { baseline }\end{array}$ & - & - & $\begin{array}{l}\text { Mother- } \\
\text { daughter } \\
\text { doing PA } \\
\text { together }\end{array}$ & $\begin{array}{c}12 \\
\text { weeks }\end{array}$ & Sig. \\
\hline 52 & $\begin{array}{l}\text { Carson et al. } \\
(2018)\end{array}$ & Canada & 8312 & $20-65$ & - & - & $\mathrm{CS}$ & Self-report & Insufficient & - & - & - & - & - \\
\hline 53 & $\begin{array}{l}\text { Mascarenhas } \\
\text { et al. (2018) }\end{array}$ & US & 64 & $\geq 18$ & $\leq 12$ & - & $\mathrm{RCT}$ & Self-report & $\begin{array}{c}\text { Insufficient PA } \\
\text { at baseline }\end{array}$ & - & - & $\begin{array}{c}\text { Video- } \\
\text { conference }\end{array}$ & $\begin{array}{c}8 \\
\text { weeks }\end{array}$ & NS \\
\hline 54 & $\begin{array}{l}\text { Carver et al. } \\
(2020)\end{array}$ & China & 322 & $\begin{array}{c}36.1 \\
( \pm 4.8)\end{array}$ & $3-5$ & $\begin{array}{c}\text { FT, PT } \\
\text { and other }\end{array}$ & $\mathrm{CS}$ & Self-report & $\begin{array}{c}18 \% \text { reported } \\
\text { not having } \\
\text { MVPA at all }\end{array}$ & $\begin{array}{l}\text { Socioecono- } \\
\text { mic status }\end{array}$ & & & & \\
\hline
\end{tabular}

Notes. PA - Physical Activity; NS - Not significant; Sig. - Significant; “_“ - no information / not specified, FT - Full Time, PT - Part Time; BS - Between subjects; CS - Cross-sectional; CSO - Cross-over; CR - Correlational; LG - Longitudinal; RCT - randomized controlled trial; R - Random/Randomized; MM - Mixed method; Qual. - Qualitative; WS - Within subjects; YR - Years. 
Figure 1. Graphical summary of the ROBIS risk assessment of bias

Figure 2. Article selection process for the current review. Based on Liberati et al. (2009)
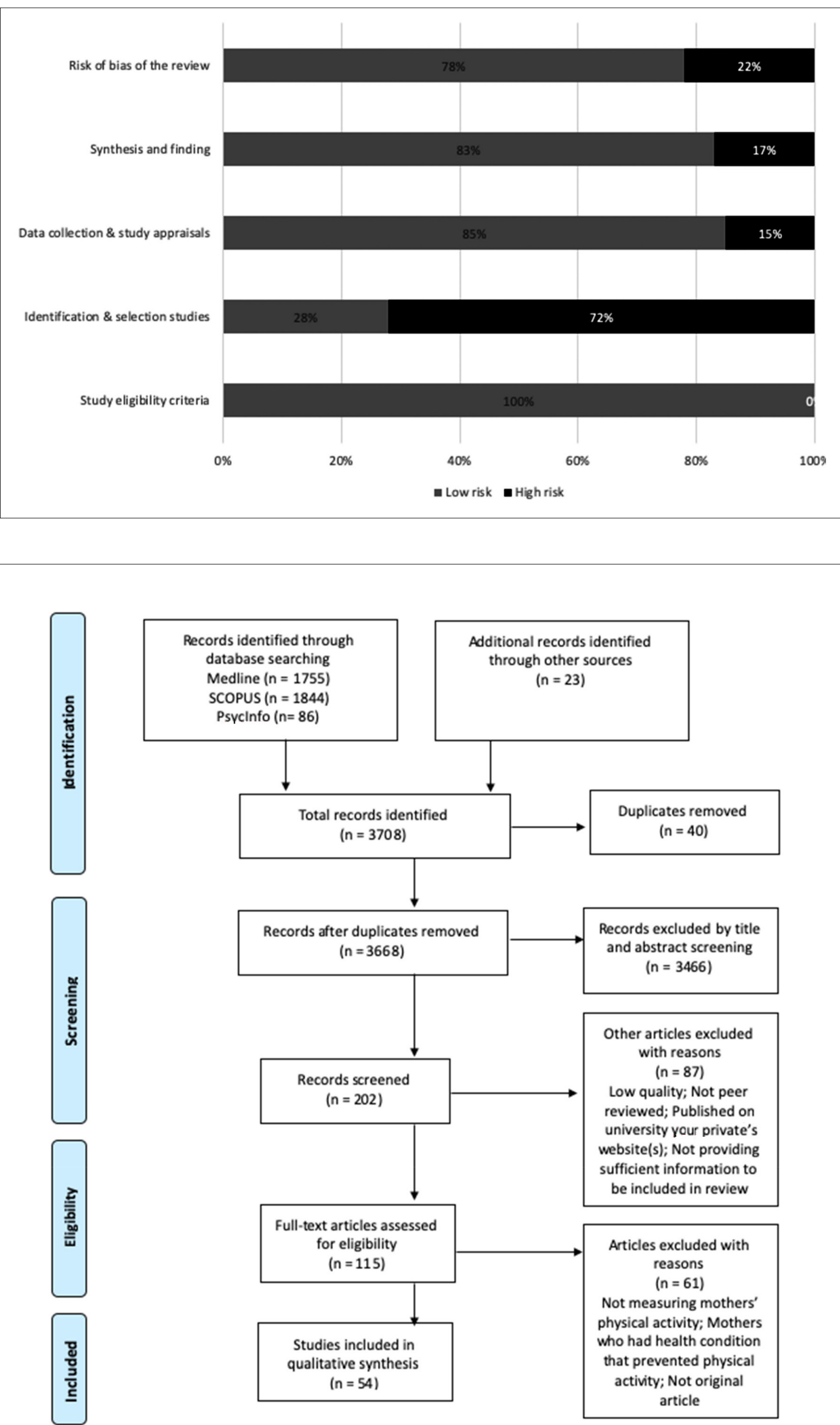

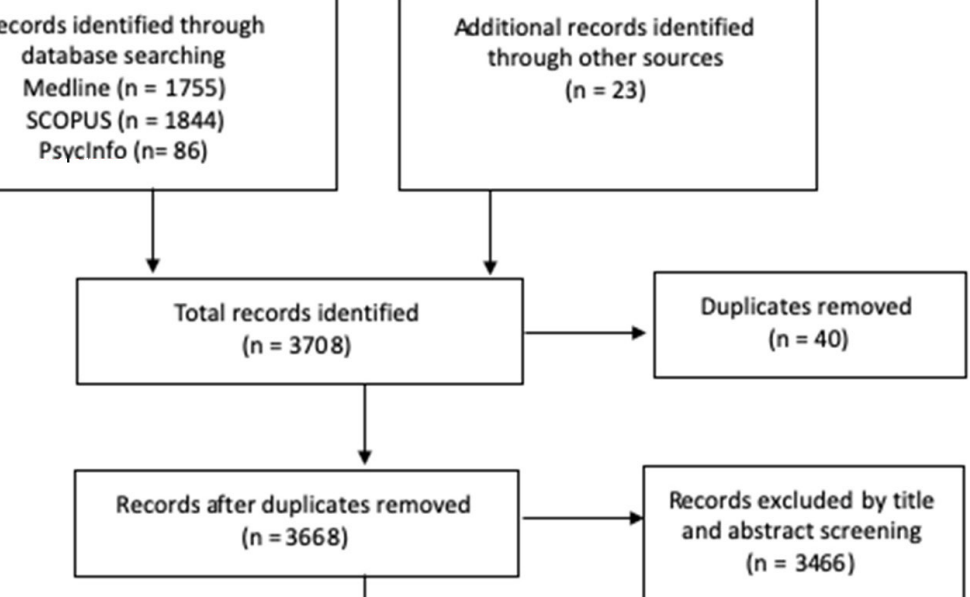

Other articles excluded with reasons

$$
\text { ( } \mathrm{n}=87 \text { ) }
$$

Low quality; Not peer reviewed; Published on university your private's website(s); Not providing sufficient information to be included in review

Articles excluded with reasons

$$
\text { ( } n=61 \text { ) }
$$

Not measuring mothers' physical activity; Mothers who had health condition that prevented physical activity; Not original article 
Each of the selected studies was evaluated in the 5 domains (24 signalling questions). Each study was rated 'yes', 'probably yes', 'probably no', 'no', or 'no Information'. The non-existence of 'no', 'probably no' and 'no Information' indicates low risk in the domain; while the presence of only one 'yes' or 'probably yes' indicates a high risk of bias in the domain. The risk of bias evaluation is shown in Table 2, and it is summarized in Figure 1.

\section{RESULTS}

Of the 54 included studies, the majority of them were conducted in western countries, with 25 studies conducted in the US. There were 14 studies conducted in Australia, 6 studies in Canada, 2 studies in Iran, and 2 other studies in China. The other five studies were in Japan, Finland, New Zealand, Poland, and United Kingdom. The studies vary in the sample size, ranging from one participant in a case study design (McGannon \& Schinke, 2013) to 8312 mothers (Carson, Adamo, \& Rhodes, 2018).

Of the included studies, 41 are quantitative studies. Nineteen of these studies employed different interventions to mothers in a variety of experimental designs: 16 studies used a randomized control trial design (Albright et al., 2012; Alhassan et al., 2018; Bronikowski et al., 2016; Clarke et al., 2007; Fjeldsoe, Miller, Graves, Barnett, \& Marshall, 2015; Jiryaee, Siadat, Zamani, \& Taleban, 2015; Keller et al., 2014; Khodabandeh et al., 2017; Lombard et al., 2009; Mailey et al., 2016; Mailey \& Hsu, 2017; Mascarenhas et al., 2018; Miller et al., 2002; Norman et al., 2010; Ransdell et al., 2003; Tuominen et al., 2017), one used a crossover design (Cramp \& Bray, 2010), and two used a withinsubjects design without a control group (Albright et al., 2009; Bronikowski et al., 2016). These studies evaluated the effectiveness of different interventions to increase mothers' PA. Of these studies, only four studies used small number of participants (e.g. $<30$ participants) (Adachi-Mejia et al., 2010; Albright et al., 2009; Bronikowski et al., 2016; Cramp \& Bray, 2010; Evenson et al., 2009; Tuominen et al., 2017), while the other studies involved a larger number of participants; seven studies used more than 100 participants, and the highest number of participants was 716.

Of the remaining 22 were quantitative studies, ten were cross-sectional (Brown et al., 2001; Candelaria et al., 2012; Carson et al., 2018; Carver et al., 2020; Dlugonski, Das, et al., 2017; Dlugonski \& Motl, 2013; Hamilton \& White, 2011; Lovell \& Butler, 2015; Olvera et al., 2011; Suzuki et al., 2017). Seven other studies employed a longitudinal study design (Bell and Lee, 2005; Dearth-Wesley et al., 2012; Evenson et al., 2009; Hull et al., 2010; Rhodes et al., 2014; Sallis et al., 2001), while three studies were correlational (Adachi-Mejia et al., 2010; Cleland et al., 2008; Dombrowski, 2011), and one was a population study (Hesketh et al., 2014). One study used an experimental design, but it did not focus on intervention effectiveness but paid attention instead to the determinants of mothers' PA (Jung \& Brawley, 2011). Four other investigations used qualitative and quantitative approaches (Albright et al., 2005; Gierc et al., 2016; Mansfield et al., 2012; Schluter et al., 2011). Overall, these studies reported mothers' PA levels, along with the barriers and facilitators of regular PA.

The remaining nine studies were qualitative. Eight out of nine qualitative studies used interviews and FGD with total participants ranging from 11 to 40 mothers (Cotter et al., 2016; Dlugonski, Martin, et al., 2017; Dlugonski \& Motl, 2016; Hamilton \& White, 2010a, 2010c; Lloyd et al., 2016; Mailey et al., 2014; Saligheh et al., 2016). Only one study used a single case study design (McGannon \& Schinke, 2013). These qualitative studies mostly tried to unravel the dynamics of different factors that determine mothers' engagement in PA.

Results of the ROBIS (Figure 1) showed that there was no risk in assessing study eligibility criteria. The authors clearly described the eligibility criteria and adhered to those criteria in screening the articles. However, there was a high risk of bias in the identification and selection of studies. This bias occurred because this review used language and publication-date delimitations and the strict PICO criteria in evaluating the eligible articles. These are delimitations that generate the risk that some pertinent studies are excluded from the review. As in most literature reviews, there was also a certain level of risk in the data collection and study appraisal stemming from the different research designs adopted in the eligible studies. The reviewed studies' heterogeneity and the applied delimitations might influence the synthesis and conclusions as estimated by a new and conservative method of bias assessment (Table 2 and Figure 1).

Mothers' physical activity level. Twentythree studies reported mothers' PA level compared to WHO guidelines for PA for adults (i.e., 150 
minutes of MVPA/week) or US CDC guidelines for postpartum mothers. The majority of these studies (17/23) were based on self-reports to assess participants PA (Albright et al., 2005; Bell \& Lee, 2005; Brown et al., 2001; Carson et al., 2018; Carver et al., 2020; Dearth-Wesley et al., 2012; Dlugonski, Martin, et al., 2017; Dombrowski, 2011; Gierc et al., 2016; Hull et al., 2010; Jiryaee et al., 2015; Lovell and Butler, 2015; Mansfield et al., 2012; Mascarenhas et al., 2018; Miller et al., 2002; Sallis et al., 2001; Suzuki et al., 2017). Five studies used objective assessment (i.e., an accelerometer during seven days) (Alhassan et al., 2018; Hesketh et al., 2014; Olvera et al., 2011; Rhodes et al., 2014; Schluter et al., 2011), and only one study used a combination of objective and self-reported measurement of PA (Albright et al., 2012).

The studies also differed in the age criteria of the participants and the age of the children. Some studies were focusing on postpartum women or mothers of infants aged under 12 months (Albright et al., 2005, 2009, 2012; Mailey \& Hsu, 2017) and three studies with participants who were mothers of preschool-aged children (Carver et al., 2020; Hesketh et al., 2014; Jiryaee et al., 2015; Sallis et al., 2001; Suzuki et al., 2017). Other studies used more lenient child's age criteria, with four inquiries requiring participants to be mothers of at least one child under the age of 10 (Alhassan et al., 2018; Dearth-Wesley et al., 2012; Gierc et al., 2016; Olvera et al., 2011) and five studies required participants to be mothers of at least one child under the age of 15 (Adachi-Mejia et al., 2010; Lovell and Butler, 2015; Mansfield et al., 2012; Mascarenhas et al., 2018; Schluter et al., 2011)

Despite different design employed, the studies included here seemed to agree that mothers do not meet PA's recommended guidelines. As a part of a national survey conducted in Canada $(n=8843)$, Carson (2018) reported that women with children in their household are significantly less likely to meet PA's recommended levels. The reported odds ratio (OR) were 0.69 [95\% Confidence Interval/CI: 056-084]. This finding is in line with other studies conducted in Australia, showing that mothers were more prone to be physically inactivity (Brown et al., 2001; Lovell \& Butler, 2015), despite smaller participants in those studies (554, 331 respectively). A recent study conducted in Hong Kong, showed that the distribution of MVPA of 322 mothers was positively skewed and that $17.7 \%$ of respondents reported having no MVPA at all (Carver, 2020).
This report indicates that mothers tend to have low PA levels. A similar conclusion was reported by Suzuki and colleagues (2017), who analysed 182 mothers, of which 46 reported some abnormalities in their most recent medical check-up. The authors found that mothers do not have enough moderate and/or vigorous PA, regardless of their current medical status. Hesketh and colleagues (2014) reported that only $53 \%$ in 554 mothers of preschool children in UK met the PA guidelines for at least one day during seven days of using an accelerometer. In their report, the average time mothers engaged in MVPA was 19 ( \pm 21.5$)$ minutes per day.

Few intervention studies included in the current review reported mothers' PA level at baseline. These studies also suggested that mothers tend not to meet the recommended guidelines. A study that used US mother and daughter dyads, Alhassan and colleagues (2018) observed that at the baseline, mothers spent on average $27.2 \pm 6.3$ minutes of light PA (LPA) in less than two days/week. Another study by Mascarenhas and colleague (2018) showed that 54 out of 64 participating mothers failed to meet the WHO's recommendation for PA at the baseline. A study conducted by Albright and colleagues (2012) revealed that the median time spent for all kinds of physical activities in 278 participating mothers was 40 minutes per week, which is way below the recommended guidelines. However, this finding is not surprising because the study excluded physically active mothers. Similarly, research with 172 Iranian mothers of children aged 1-5 years showed that most mothers reported low PA levels before an intervention (Jiryaee et al., 2015).

Three longitudinal studies indicated that transition into motherhood significantly impacts PA level. Bell and Lee (2005) tracked more than 8000 women in 4 years. Their analysis showed that women who became mothers over the study period were 1.6 more likely to decrease their level of PA than those who did not give birth to a child in the same period. Hull and colleagues (2010) revealed similar findings by following 349 women over two years. While there was no significant difference in PA level between participants who did not give birth and those who had their first child during the study period, the latter participants showed a marked decrease in PA compared to the former group. The effect size of the change in the PA level was moderate $(d=0.52)$. Rhodes and colleagues (2014) assessed 238 married couples on three different occasions between 2007-2011. There 
was no difference in MVPA between participants with children and without children during the baseline. However, first-time mothers had the most significant decrease in MVPA across a 12-months study period. Another study also suggested that motherhood impacts the PA level (Albright et al., 2005). In this study, the authors asked 79 postpartum participants, retrospectively, about their current and prior-to pregnancy PA. The results indicated that a significant proportion fell into the "Active prior Pregnancy-Inactive postpartum" group (43\%), suggesting that transition into motherhood harmed the regular PA habit.

Although studies suggest that mothers tend to be less active and that mothers are more prone to physical inactivity than non-mothers, a different pattern was reported by Sallis and colleagues (2001). Their seven-year longitudinal study of 226 American mothers' disclosed a significant increase in both moderate and vigorous leisure PA. Further, their analysis refuted that PA level increase is related to having their previously preschool-age children grow up (hence providing more leisure time to exercise for mothers). Another study by Candelaria and colleagues (2012) also produced a similar result. Their design involved both objective and self-report measures of PA. The analysis of 909 mothers and non-mothers revealed that there was no significant difference in the objectively measured PA and both groups accrue PA that is slightly below the recommended 30 minutes/day, five days a week. However, the self-report assessment showed that mothers reported significantly higher PA related to household chores compared to women without children.

Barriers or facilitators of physical activity engagement in mothers. Twenty included studies investigated correlates of mothers' engagement in PA. A study examining 1691 US mothers showed that lack of time, lack of self-discipline, and lack of energy were the three most often cited reasons for not being active (Adachi-Mejia et al., 2010). In another study of 2244 New Zealand and Pacific Island mothers, a) "lack of time due to family responsibilities", b) "I would have to get someone to get my children," and c) "I have too many household chores to do" were the three perceived barriers with the highest median scores (Schluter et al., 2011). These reasons are all tied to household responsibilities. This inference of mothers is due to more family and household responsibilities, and hence the inability to become physically active as resonated in other studies as well (Dlugonski \& Motl, 2016; Evenson et al., 2009; Mailey et al., 2014; McGannon \& Schinke, 2013; Saligheh et al., 2016). Most mothers in the examined studies were primary caregivers of the children, which might have prevented them from engaging in PA. They reported guilt if they engaged in PA because they prioritized their own need before their family's needs (Lloyd et al., 2016; Mailey et al., 2014; McGannon \& Schinke, 2013).

The role as a primary caregiver for children was also related to scheduling constraints; as mothers prioritize family and specifically childcare, they reported having difficulties in arranging their schedule to integrate PA (Dlugonski \& Motl, 2016; Evenson et al., 2009; Hamilton \& White, 2010c; Mailey et al., 2014; McGannon \& Schinke, 2013; Schluter et al., 2011). These results match the finding of a study adopting the theories of planned behaviour that the fear of clash with other commitments (e.g., commitment to take care of children), lack of motivation, and lack of time could be predictors of mothers' PA in a negative direction (Hamilton \& White, 2011).

Additional responsibilities of mothers seemed to play a role in mothers' decreasing PA. Scheduling was even more difficult for mothers who had a full-time job, which increased their already broad range of responsibilities (Hamilton \& White, 2011; Mailey et al., 2014; McGannon \& Schinke, 2013). Even if they managed to integrate PA into their daily schedules, there were days when they felt too tired or had no energy to exercise (Adachi-Mejia et al., 2010; Dlugonski \& Motl, 2016; Evenson et al., 2009; Schluter et al., 2011).

Another factor that can serve as either facilitator or barrier is self-efficacy. Mothers reported a lack of self-efficacy to engage in PA suitable for their circumstances (Dlugonski, Das, et al., 2017; Dombrowski, 2011). Mothers who believed that they could do PA (self-efficacy) or fulfil different roles (concurrent self-regulatory efficacy) were more likely to engage in PA regularly (Dombrowski, 2011; Jung \& Brawley, 2011). Some studies also showed that having or not having social support from the family could be a facilitator or barrier, depending on the situation. A spouse who is encouraging the mother to exercise or willing to help with the childcare is an essential facilitator of PA, as well as having family members or friends who can help in childcare (Albright et al., 2005; Cotter et al., 2016; Dlugonski, Das, et al., 2017; Dlugonski \& Motl, 
2016; Mailey et al., 2014; McGannon \& Schinke, 2013; Saligheh et al., 2016). In one correlational study, social support was among the critical facilitators for mothers willing to engage in exercise. Indeed, social support emerged as a significant PA predictor among mothers (Dlugonski, Das, et al., 2017). This finding agrees with qualitative studies showing that spousal support is one of the key facilitators for active mothers (Cotter et al., 2016; Hamilton \& White, 2010c; Mailey et al., 2014). On the downside, a spouse supporting the mother to engage in PA but is unwilling to share in the family responsibilities while the mother exercises is a barrier. A few studies reported that spouses often encourage mothers to exercise on conditional terms (i.e., without taking over some of the mothers' responsibilities) (Cotter et al., 2016; Mailey et al., 2014; McGannon \& Schinke, 2013).

Another motive that drives mothers to be physically active is the desire to be a role model for their children (Hamilton \& White, 2010c; Lloyd et al., 2016; Mailey et al., 2014; Schluter et al., 2011). Mothers would also engage in PA more if they could see the benefits of such activities on the family's overall physical and mental health (Hamilton \& White, 2010a, 2010c; Lloyd et al., 2016; McGannon $\&$ Schinke, 2013).

Sociodemographic variables (e.g., income or financial constraints) and environmental factors (neighborhood safety, lack of public facilities, lack of community support) are also barriers to mothers' PA. Mothers' perception of the quality of local facilities and social environment was associated with more persistent leisure-time walking in 357 mothers (Cleland et al., 2008). A recent study by Carver and colleagues (2020) revealed that numerous socioeconomic variables indirectly impact mothers' PA in complex pathways. These include mothers' education level, employment status (full-time), and income. All three were positively associated with having a helper, allowing mothers to have more leisure time to exercise. Although sociodemographic and environmental factors influence mothers' PA, these variables are less influential than intra- and interpersonal variables (Cotter et al., 2016; Evenson et al., 2009; Saligheh et al., 2016).

Efficacy of the interventions aimed at increasing mothers' physical activity. Social support intervention is the most common area targeted in the included studies. However, the operationalization of the intervention might differ between studies. Keller and colleagues (2014) evaluated a 12 months' intervention focusing on the impact of providing social support to PA level in 139 obese and overweight Latin mothers using a randomized control trial. Their results showed that mothers in the intervention group spent significantly more time to walk more steps than controls at 6 and 12 months and accrued more aerobic steps.

Few studies operationalized social support by devising intervention allowing mothers and children to exercises together. Ransdell and colleagues (2003) studied 34 dyads of mothers and their teenage daughters. This communitybased intervention aimed to improve or worsen the PA level of these dyads compared to home-based intervention. Their results showed that mothers in both groups significantly increased their aerobic activity participation, muscle-strengthening activity, and flexibility-focused activity. They also reported a large effect size of both community and home-based intervention to these changes. No difference was evident among the two groups. Alhassan and colleagues (2018) studied 76 AfricanAmerican mothers and daughter dyads. Their intervention was based on social cognitive theory and addressed the issue of self-efficacy in mothers. The result showed that mothers who were required to be physically active together with their daughters showed a significant increase after 12-weeks intervention compared to mothers who were offered to exercise independently of their daughter.

Although the above studies suggest that exercising together with children could positively impact mothers' PA, another study by Norman and colleagues (2010) showed different results. The authors randomized 135 primiparous and multiparous mothers into one of the two conditions: a) an intervention group in which the participants were required to exercise with their babies, and b) an education-only group. After 8-weeks of intervention, there were no differences in PA levels between the groups, but the mothers in mother and baby condition reported significant improvement in well-being. This result is like that reported by Bronikowski and colleagues (2016), evaluating how mothers and children exercising together impact mothers' PA level during a 15-week intervention. Their analysis showed no change in the 14 mothers' MVPA days/week before and after the intervention. Tuominen and colleagues (2016) compared mothers who danced with their children $(n=11)$ and a control group $(n=13)$ during a 2 -week 
intervention. Their findings showed that both groups reported higher MVPA in the post-test than in the pre-test measurement. Still, this increase was not statistically significant, and no between-groups difference was evident. Therefore, it appeared that exercising with children does not affect mothers' PA level.

Other studies focused on how social support is delivered during the intervention period and adjusted to different individuals. Fjeldsoe and colleagues (2015) compared the impact of an intervention comprising face-to-face and telephone counselling and tailored support text messages ( $\mathrm{n}=130$ mothers) to mothers' objective and selfreport PA. Their study revealed that mothers in the intervention group reported that they engaged in 1.6 more days and 48.5 minutes more than the control group, which was statistically significant. However, objective data from accelerometers indicated that no significant difference existed between the groups. Similarly, Khodabandeh and colleagues (2017) conducted a randomized control trial on 220 postpartum mothers. In the intervention group, mothers received face-to-face, phone, and text messages as counselling and a booklet in addition to routine postpartum training. After six weeks of intervention, no difference in moderate PA was observed, with mothers in the intervention arm reporting an average of $16 \pm 15.2$ minutes while control participants reported only $8 \pm 7.9$ minutes.

Other areas of interest for intervention revolve around problem-solving ability, goal setting, and self-efficacy to overcome PA barriers. An Iranian study (Jiryaeeet al., 2015) on 172 mothers of children aged 1-5 years studied the effects of group-based goal-setting on PA levels. Results revealed that mothers who received the intervention showed a significant increase in their PA score compared to control participants post intervention, and two months after the intervention. Lombard and colleagues (2009) conducted a Randomized Control Trial (RCT) to compare the impact of an interactive intervention that addressed problemsolving, goal-setting, self-monitoring, and relapse prevention to non-interactive health education sessions. Their finding unveiled that both groups increased PA. However, although the intervention reported more significant PA increases, the difference between groups was statistically not significant. Mailey and Hsu (2017) showed that an intervention that facilitates mothers' self-regulatory skills and efficacy in dealing with PA barriers could increase the self-reported level of PA. The authors tested 49 participants randomly assigned to general recommendation intervention or specific recommendation intervention. There was a large increase in the self-reported PA in both groups. Further analysis revealed that recommending a general PA level produced a more considerable change than a specific PA level recommendation.

With the growing use of the internet, few studies evaluated the feasibility of internet-based delivery of the intervention to mothers' PA. Mailey and colleagues (2016) devised a study to compare an 8-week standard web-based intervention to a self-determination theory-guided intervention in 69 mothers. They found that both groups reported a significant increase in PA from baseline to week 8 (end of intervention). Mascarenhas and colleagues (2018) evaluated the efficacy of an intervention delivered through a mobile application on mothers' PA. Although their finding showed that mothers in the intervention arm $(n=29)$ increased their MVPA and MPA minutes per week by an average of 42.2 minutes and 13.8 minutes, respectively, the difference between the two groups was not significant.

Clarke and colleagues (2007) studied the impact of providing a pedometer and 8 weekly lessons of recommendations for PA, healthful eating, and behavior modification for 93 mothers compared to 31 control participants who did not receive a pedometer. Their finding suggests that participants in the experimental group reported significantly more steps/day after eight weeks.

\section{DISCUSSION}

This review's first objective was to examine whether mothers meet the international guidelines for PA (WHO and US CDC). This review shows that despite differences in the research methods used, mothers do not meet the guidelines for PA for health (Albright et al., 2005; Brown et al., 2001; Carson et al., 2018; Dlugonski, Martin, et al., 2017; Dombrowski, 2011; Gierc et al., 2016; Jiryaee et al., 2015; Lovell \& Butler, 2015; Mailey \& McAuley, 2014; Mascarenhas et al., 2018; Miller et al., 2002). Unfortunately, this finding matches the conclusion of the past literature review by Bellows-Riecken and Rhodes (2008) ten years earlier and worldwide trends in PA from 2001-2016 (Guthold et al., 2018). Consequently, insufficient PA continues to be a potential health hazard for women with children. 
Insufficient PA is noted in mothers of new-borns and infants (Albright et al., 2005; Evenson et al., 2009; Keller et al., 2014), toddlers (Hesketh et al., 2014; Jiryaee et al., 2015; McGannon \& Schinke, 2013; Sallis et al., 2001), and school-aged children (Cleland et al., 2008; Dearth-Wesley et al., 2012; Dlugonski \& Motl, 2013; Gierc et al., 2016; Jung \& Brawley, 2011; Lovell \& Butler, 2015; Mailey et al., 2014; Schluter et al., 2011). At the first glance, these findings might suggest that the child's age is not related to the mothers' PA level (Candelaria et al., 2012; Sallis et al., 2001). However, more research is needed on the family structure (number and age of children) to establish the effect of a child's age on mothers' PA.

The current review also reveals that transition into motherhood could harm mothers' PA level (Bell \& Lee, 2005; Hull et al., 2010; Rhodes et al., 2014). However, at least one study (Candelaria et al., 2012) in the current review revealed that differences between the groups only occurred in leisure type of physical activities. Therefore, mothers may get enough PA from non-leisurely movements accumulated throughout the day, which is a hypothesis that merits further research consideration. Future longitudinal studies should investigate the differences in more specific domains of PA (context of the PA: work-related, transportation, housework, and leisure $\mathrm{PA}$; or intensity level of the PA: light, moderate-tovigorous, vigorous) to gather a clearer picture about the domain of PA that would be the most suitable for mothers.

Another objective of the review was to examine the barriers and facilitators of PA in mothers. This review revealed that personal, social, and physical environmental factors could be determinants of the mothers' PA participation. Mothers often assume the role of primary childcare provider in the household, which may lead to guilt if they prioritize PA over other responsibilities (Lloyd et al., 2016; Mailey et al., 2014; McGannon \& Schinke, 2013). Mothers also report difficulties in managing multiple roles (housework and child care provision, work obligations, and the need to take care of themselves) (Adachi-Mejia et al., 2010; Albright et al., 2005; Cotter et al., 2016; Evenson et al., 2009; Mailey et al., 2014; Mansfield et al., 2012; Saligheh et al., 2016; Schluter et al., 2011). Having support from the spouse or close family can be a pivotal factor for mothers to engage in regular PA (Albright et al., 2005, 2012; Cleland et al., 2008; Cotter et al.,
2016; Mailey et al., 2014; McGannon \& Schinke, 2013; Miller et al., 2002). Whether it is help with the childcare or house chores or simple encouragement to exercise from significant others, all can facilitate mothers' regular exercise participation.

Becoming a mother can also be an incentive for a healthier lifestyle and regular PA. Indeed, a few studies reported that mothers used PA to become role models for their children (Dlugonski, Martin, et al., 2017; Dlugonski \& Motl, 2016; Mailey et al., 2014; Schluter et al., 2011). However, this incentive is often thwarted by a lack of knowledge or strategies to exercise properly. In this case, having community support or enrolment in a structured PA program could increase the mothers' PA level (Cleland et al., 2008; Dlugonski, Das, et al., 2017; Saligheh et al., 2016). Another way to motivate PA for the new mothers is by focusing on the outcome expectancy or perceived benefit. Those who believed that sufficient PA would enable them to cope with multiple roles and demands as mothers often showed an intention to engage in regular PA (Rhodes et al., 2014). Exercising also provided mothers a 'time for me' necessary for dealing with a variety of self-concern issues.

Other than personal and social factors, few other variables also played a part in mothers' PA. Low household income and lack of access to exercise facilities were barriers to mothers' PA, especially if they come from a low socioeconomic class (Cotter et al., 2016; Dlugonski, Martin, et al., 2017). The urgency to pay for living expenses can make mothers work double shifts and limit their time and energy to engage in scheduled exercise. Neighbourhood safety and community support also influences mothers' engagement in physical activities (Cleland et al., 2008; Mansfield et al., 2012). If the neighbourhood did not support PA and the perceived safety was low, mothers' chances to do moderate-to-vigorous PA were also small. However, the effect of these socioeconomic and environmental variables on PA is not direct, and as Mailey and Hsu (2017) suggest, complex pathways exist among them.

Despite the fact that a great deal of research has been conducted on PA's correlates in mothers, the studies have primarily focused only on one or two domains. Future research with a more integrative and holistic view of the correlations is needed to understand better the interactions between the various factors that affect mothers' regular PA. The use of a theoretical framework in understanding 
how different factors mediate PA should be adopted (Davis et al., 2014). The use of a theoretical framework would then guide the devising of effective interventions. Future research also needs to investigate sociodemographic, personal, social, cultural, and environmental factors to understand PA patterns in mothers better.

This review's last objective was to examine the effectiveness of interventions aimed at increasing mothers' PA. Findings suggest that interventions often target psychological variables such as goal setting, problem solving, self-efficacy, and social support. Intervention that targets these variables seemed to have a higher impact on mothers' PA, especially compared to interventions that merely target mothers' knowledge about PA's health benefits (Keller et al., 2014; Khodabandeh et al., 2017).

It seems that effective interventions in increasing mothers' PA used combinations of individual counselling, facilitated group discussions to set PA goals, monitoring of the progress, group discussion about overcoming barriers, as well as professionally guided exercise sessions (Albright et al., 2009; Jiryaee et al., 2015; Mailey et al., 2016; Mailey \& McAuley, 2014; Miller et al., 2002; Ransdell et al., 2003). These approaches essentially targeted psychological variables such as selfefficacy and thought barriers to regular exercise. Although the interventions and their duration varied significantly, their results are promising. Some suggest that effective interventions should be tailored to participants' conditions (Fjeldsoe et al., 2015; Khodabandeh et al., 2017).

Although the result of interventions targeting psychological variables seemed promising, it should be interpreted with caution because the reported change in the PA of the participants in an exercise intervention might be due (at least in part) to their participation in the research. This is a phenomenon related to demand characteristics known as the 'Hawthorne effect.' Few studies concluded that by asking participants to recall their PA over the last seven days or by simply providing them with an accelerometer, they made participants aware that they are monitored and alters the behavior (Clarke et al., 2007; Fjeldsoe et al., 2015).

Interventions that examined the impact of exercising together with the child as part of social support showed mixed results. While it seemed that this type of intervention is effective for studies with mothers of teenagers from low socioeconomic status (Alhassan et al., 2018; Ransdell et al., 2003), more systematic research is needed with mothers of babies (Norman et al., 2010), preschool children (Tuominen et al., 2016), and teenagers from middle and high socioeconomic status (Bronikowski et al., 2016). Despite the mixed results, these studies reported that exercising together with their children might result in mothers feeling supported and providing a role model to the child/children.

The intervention studies included in the current review were lack spousal support. Most studies did not monitor social/spousal support in the intervention period. Given that partners' support could affect mothers' exercise behaviour, future research should also consider integrating spouse/ partner to exercise together as the interventions mentioned above did not involve husband/partner in their study. Exercising together with a spouse or discussing how household responsibilities are divided to enable mothers to have leisure time might impact mothers' PA.

With the growing advancement and use of technology for daily purposes, few studies have tested the feasibility of using the application- and web-based programs to increase the mothers' PA level (Fjeldsoe et al., 2015; Mailey et al., 2016; Mascarenhas et al., 2018; Tuominen et al., 2016). Although these intervention results appear to be promising, more research is needed especially on the long-term use of the technology and adherence to their use in self-managed or organized PA programs.

This current review has limitations too The first was the number of databases used. Only three major databases were used in this review, hence limiting the number of articles. The second limitation revolves around the broad conceptualization of PA. The bulk of the articles included in the current review measured a general category of $P A$, while there exist more specific categories for PA (i.e., leisure time PA, work-related PA, commuting PA, household PA). Analyzing the PA behavior in such detailed manner will lead to better understanding of mothers' PA: how physically active are the mothers in these various sub-domains? Understanding specific patterns of PA could lead to a more effective intervention in the future. Another limitation, like in many other systematic reviews, is applying language and publication date limitation. The use of language delimitation might have resulted in more studies conducted in US and other high-income Western 
countries (e.g. UK, Australia) that could be included in this review. This delimitation might have biased the current review results, considering that the prevalence of physical inactivity in women varies in different world regions (Guthold et al., 2018). Despite these limitations, the current review has at least twice as many studies included than in the two earlier reviews published in the new millennium (Abbasi \& van den Akker, 2015; Bellows-Riecken $\&$ Rhodes, 2008) and it includes the largest number of intervention studies ever included in a literature review on the topic.

\section{CONCLUSIONS}

Transition into motherhood seems to have a significant negative impact on PA. Several barriers associated with motherhood hinder mothers from being physically active. While interventions to increase PA in mothers increase and yield promising results, their cessation frequently leads to relapse in inactivity. Results suggest that interventions should enhance mothers' selfefficacy, goal setting, problem-solving, social support, and knowledge about PA to be effective. More research is needed by using interventions that involve children and spouses. The longterm benefits of smartphone, or internet-based applications, in motivating mothers' PA should also be explored. This review's key message is that compared to the other two reviews published earlier in the new millennium, there are no significant changes in mothers' PA levels. Slightly promising results emerged from intervention research that should use more post-intervention (longitudinal) measurements to determine the facilitating and debilitating effects of environmental and social factors in adherence to exercise. The surfacing of technology-based applications aimed at promoting PA is also a challenging subject of future research. It can be envisaged that interventions, new modern technology-support, and a more significant share of parenting may lead to a more optimistic outcome in a future literature review in the area.

Nevertheless, before drawing a dim conclusion, these authors draw the readers' attention to the conjecture that mothers may have more PA during their home chores and childcare and at homework than those who report PA levels matching/exceeding the WHO's guidelines US CDC. Consequently, a major challenge facing future research is to untangle the physical activities performed in various subdomains of life in drawing a conclusion about mothers' PA level. The recently available accelerometers and smartwatches are promising tools in elucidating this conjecture.

\section{REFERENCES}

Abbasi, M., \& van den Akker, O. (2015). A systematic review of changes in women's physical activity before and during pregnancy and the postnatal period. Journal of Reproductive and Infant Psychology, 33(4), 325-358. https://doi.org/10.1080/02646838.2015.1012710

Adachi-Mejia, A. M., Drake, K. M., Mackenzie, T. A., Titus-ernstoff, L., Longacre, M. R., Hendricks, K. M., Beach, M. L., \& Dalton, M. A. (2010). Perceived intrinsic barriers to physical activity among rural mothers. Journal of Women's Health, 19(12), 2197-2202. https:// doi.org/10.1089/jwh.2009.1879

Albright, C. L., Maddock, J. E., \& Nigg, C. R. (2009). Increasing physical activity in postpartum multiethnic women in Hawaii: Results from a pilot study. $B M C$ Women's Health, 9, 1-7. https://doi.org/10.1186/14726874-9-4

Albright, C. L., Maddock, J. E., \& Nigg, C. R. (2005). Physical activity before pregnancy and following childbirth in a multiethnic sample of healthy women in Hawaii. Women and Health, 42(3), 95-110. https://doi. org/10.1300/J013v42n03_06

Albright, C. L., Steffen, A. D., Novotny, R., Nigg, C. R., Wilkens, L. R., Saiki, K., Yamada, P.,
Hedemark, B., Maddock, J. E., Dunn, A. L., \& Brown, W. J. (2012). Baseline results from Hawaii's Nā Mikimiki Project: A physical activity intervention tailored to multiethnic postpartum women. Women and Health, 52(3), 265-291. https://doi.org/10.1080/03630242.2012.662935

Alhassan, S., Nwaokelemeh, O., Greever, C. J., Burkart, D. J. (2018). Effect of a culturally-tailored mother-daughter physical activity intervention on pre-adolescent African-American girls' physical activity levels. Preventive Medicine Reports, 11 (November 2017), 7-14. https://doi.org/10.1016/j. pmedr.2018.05.009

Archer, E., Lavie, C. J., McDonald, S. M., Thomas, D. M., Hébert, J. R., Taverno Ross, S. E., McIver, K. L., Malina, R. M., \& Blair, S. N. (2013). Maternal inactivity: 45-year trends in mothers' use of time. Mayo Clinic Proceedings, 88(12), 1368-1377. https://doi.org/10.1016/j.mayocp.2013.09.009

Bell, S., \& Lee, C. (2005). Emerging Adulthood and Patterns of Physical Activity Among Young Australian Women. International Journal of Behavioral Medicine, 12(4, 3), 227-235. https://doi.org/10.1207/ s15327558ijbm1303 11 
Bellows-Riecken, K. H., \& Rhodes, R. E. (2008). A birth of inactivity? A review of physical activity and parenthood. Preventive Medicine, 46(2), 99-110. https:// doi.org/10.1016/j.ypmed.2007.08.003

Bronikowski, M., Bronikowska, M., Pluta, B., Maciaszek, J., Tomczak, M., \& Glapa, A. (2016). Positive impact on physical activity and health behaviour changes of a 15-week family focused intervention program: "Juniors for Seniors". BioMed Research International, 2016, 1-8. https://doi.org/10.1155/2016/5489348

Brown, P. R., Brown, W. J., Miller, Y. D., \& Hansen, V. (2001). Perceived constraints and social support for active leisure among mothers with young children. Leisure Sciences, 23(3), 131-144. https://doi. org/10.1080/014904001316896837

Candelaria, J. I., Sallis, J. F., Conway, T. L., Saelens, B. E., Frank, L. D., \& Slymen, D. J. (2012). Differences in physical activity among adults in households with and without children. Journal of Physical Activity and Health, 9(7), 985-995. https://doi.org/10.1123/jpah.9.7.985

Carson, V., Adamo, K., \& Rhodes, R. E. (2018). Associations of parenthood with physical activity, sedentary behavior, and sleep. American Journal of Health Behavior, 42(3), 80-89. https://doi.org/10.5993/ AJHB.42.3.8

Carver, A., Akram, M., Barnett, A., Mellecker, R., \& Cerin, E. (2020). Socioeconomic status and physical activity among mothers of young children in an asian city: The mediating role of household activities and domestic help. International Journal of Environmental Research and Public Health, 17(7), 1-16. https://doi. org/10.3390/ijerph17072498

Clarke, K. K., Freeland-Graves, J., KloheLehman, D. M., Milani, T. J., Nuss, H. J., \& Laffrey, S. (2007). Promotion of physical activity in low-income mothers using pedometers. Journal of the American Dietetic Association, 107(6), 962-967. https://doi. org/10.1016/j.jada.2007.03.010

Cleland, V. J., Timperio, A., \& Crawford, D. (2008). Are perceptions of the physical and social environment associated with mothers' walking for leisure and for transport? Alongitudinal study. Preventive Medicine, 47(2), 188-193. https://doi.org/10.1016/j.ypmed.2008.05.010

Cotter, E. W., Hamilton, N. S., Kelly, N. R., Harney, M. B., Greene, L. S., White, K. A., \& Mazzeo, S. E. (2016). A Qualitative examination of health barriers and facilitators among African American mothers in a subsidized housing community. Health Promotion Practice, 17(5), 682-692. https://doi.org/10.1177/1524839916630504

Cramp, A. G., \& Bray, S. R. (2010). Postnatal women's feeling state responses to exercise with and without baby. Maternal and Child Health Journal, 14(3), 343-349. https://doi.org/10.1007/s10995-009-0462-5

Dearth-Wesley, T., Gordon-Larsen, P., Adair, L. S., Zhang, B., \& Popkin, B. M. (2012). Longitudinal, cross-cohort comparison of physical activity patterns in Chinese mothers and children. International Journal of Behavioral Nutrition and Physical Activity, 9, 1-9. https://doi.org/10.1186/1479-5868-9-39
Dlugonski, D., Das, B. M., Martin, T. R., \& Palmer, A. (2017). Collective efficacy, physical activity, and health outcomes among mothers. Family and Community Health, 40(4), 316-323. https://doi.org/10.1097/ FCH.0000000000000162

Dlugonski, D., Martin, T. R., Mailey, E. L., \& Pineda, E. (2017). Motives and barriers for physical activity among low-income black single mothers. Sex Roles, 77, 379392. https://doi.org/10.1007/s11199-016-0718-7

Dlugonski, D., \& Motl, R. W. (2013). Marital status and motherhood: Implications for physical activity. Women and Health, 53(2), 203-215. https://doi.org/10.1080/03 630242.2013.767304

Dlugonski, D., \& Motl, R. W. (2016). Physical activity experiences and beliefs among single mothers: A qualitative study. Research Quarterly for Exercise and Sport, 87(3), 311-317. https://doi.org/10.1080/0270136 7.2016.1187705

Dombrowski, J. J. (2011). Barriers to physical activity among working mothers. AAOHN Journal: Official Journal of the American Association of Occupational Health Nurses, 59(4), 161-167. https://doi. org/10.3928/08910162-20110328-02

Evenson, K. R., Aytur, S. A., \& Borodulin, K. (2009). Physical activity beliefs, barriers, and enablers among postpartum women. Diabetic Medicine, 16(9), 793-796. https://doi.org/10.1089/jwh.2008.1309

Fjeldsoe, B. S., Miller, Y. D., Graves, N., Barnett, A. G. \& Marshall, A. L. (2015). Randomized controlled trial of an improved version of mobilemums, an intervention for increasing physical activity in women with young children. Annals of Behavioral Medicine, 49(4), 487499. https://doi.org/10.1007/s12160-014-9675-y

Gaston, A., Edwards, S. A., Doelman, A., \& Tober, J. A. (2014). The impact of parenthood on Canadians' objectively measured physical activity: An examination of cross-sectional population-based data. BMC Public Health, 14, 1127. https://doi.org/http://dx.doi. org/10.1186/1471-2458-14-1127

Gierc, M., Locke, S., Jung, M., \& Brawley, L. (2016). Attempting to be active: Self-efficacy and barrier limitation differentiate activity levels of working mothers. Journal of Health Psychology, 21(7), 13511360. https://doi.org/10.1177/1359105314553047

Guthold, R., Stevens, G. A., Riley, L. M., \& Bull, F. C. (2018). Worldwide trends in insufficient physical activity from 2001 to 2016: A pooled analysis of 358 population-based surveys with 1.9 million participants. The Lancet Global Health, 6(10), e1077-e1086. https:// doi.org/10.1016/S2214-109X(18)30357-7

Hamilton, K., \& White, K. M. (2011). Identifying key belief-based targets for promoting regular physical activity among mothers and fathers with young children. Journal of Science and Medicine in Sport, 14(2), 135142. https://doi.org/10.1016/j.jsams.2010.07.004

Hamilton, K., \& White, K. M. (2010a). Identifying parents perceptions about physical activity: A qualitative exploration of salient behavioural, normative and control beliefs among mothers and fathers of young children. 
Journal of Health Psychology, 15(8), 1157-1169. https:// doi.org/10.1177/1359105310364176

Hamilton, K., \& White, K. M. (2010b). Parental physical activity: Exploring the role of social support. American Journal of Health Behavior, 34(5), 573-584. https://doi. org/10.5993/AJHB.34.5.7

Hamilton, K., \& White, K. M. (2010c). Understanding parental physical activity: Meanings, habits, and social role influence. Psychology of Sport and Exercise, 11(4), 275-285. https://doi.org/10.1016/j. psychsport.2010.02.006

Hartman, M. A., Hosper, K., \& Stronks, K. (2011). Targeting physical activity and nutrition interventions towards mothers with young children: A review on components that contribute to attendance and effectiveness. Public Health Nutrition, 14(8), 13641381. https://doi.org/10.1017/S1368980010001941

Hesketh, K. R., Goodfellow, L., Ekelund, U., McMinn, A. M., Godfrey, K. M., Inskip, H. M., Cooper, C., Harvey, N. C., \& van Sluijs, E. M. F. (2014). Activity levels in mothers and their preschool children. Pediatrics, 133(4), e973-e980. https://doi.org/10.1542/ peds.2013-3153

Hull, E. E., Rofey, D. L., Robertson, R. J., Nagle, E. F., Otto, A. D., \& Aaron, D. J. (2010). Influence of marriage and parenthood on physical activity: A 2-year prospective analysis. Journal of Physical Activity and Health, 7(5), 577-583. https://doi.org/10.1123/jpah.7.5.577

Jiryaee, N., Siadat, Z. D., Zamani, A., \& Taleban, R. (2015). Comparing of goal setting strategy with group education method to increase physical activity level: A randomized trial. Journal of Research in Medical Sciences, 20(10), 987-993. https://doi.org/10.4103/17351995.172792

Jung, M. E., \& Brawley, L. R. (2013). Concurrent self-regulatory efficacy as a mediator of the goal: Exercise behaviour relationship. Journal of Health Psychology, 18(5), 601-611. https://doi. org/10.1177/1359105313479238

Jung, M. E., \& Brawley, L. R. (2011). Exercise persistence in the face of varying exercise challenges: A test of self-efficacy theory in working mothers. Journal of Health Psychology, 16(5), 728-738. https://doi. org/10.1177/1359105310388322

Keller, C., Ainsworth, B., Records, K., Todd, M., Belyea, M., Vega-López, S., Permana, P., Coonrod, D., \& Nagle-Williams, A. (2014). A comparison of a social support physical activity intervention in weight management among postpartum Latinas. BMC Public Health, 14(1). https://doi.org/10.1186/1471-2458-14-971

Khodabandeh, F., Mirghafourvand, M., Kamalifard, M., Mohammad-Alizadeh-Charandabi, S., \& Asghari Jafarabadi, M. (2017). Effect of educational package on lifestyle of primiparous mothers during postpartum period: A randomized controlled clinical trial. Health Education Research, 32(5), 399-411. https://doi. org/10.1093/her/cyx060

Li, K., Davison, K. K., \& Jurkowski, J. M. (2012). Mental health and family functioning as correlates of a sedentary lifestyle among low-income women with young children. Women and Health, 52(6), 606-619. https://doi.org/10.1080/03630242.2012.705243

Liberati, A., Altman, D. G., Tetzlaff, J., Mulrow, C., Gøtzsche, P. C., Ioannidis, J. P. A., Clarke, M., Devereaux, P. J., Kleijnen, J., \& Moher, D. (2009). The PRISMA statement for reporting systematic reviews and meta-analyses of studies that evaluate health care interventions: Explanation and elaboration. Journal of Clinical Epidemiology, 62(10), e1-34. https://doi. org/10.1016/j.jclinepi.2009.06.006

Lloyd, K., O’Brien, W., \& Riot, C. (2016). Mothers with young children: Caring for the self through the physical activity space. Leisure Sciences, 38(2), 85-99. https:// doi.org/10.1080/01490400.2015.1076362

Lombard, C. B., Deeks, A. A., Ball, K., Jolley, D., \& Teede, H. J. (2009). Weight, physical activity and dietary behavior change in young mothers: Short term results of the HeLP-her cluster randomized controlled trial. Nutrition Journal, 8(1), 1-9. https://doi. org/10.1186/1475-2891-8-17

Lovell, G. P., \& Butler, F. R. (2015). Physical activity behavior and role overload in mothers. Health Care for Women International, 36(3), 342-355. https://doi.org/10. 1080/07399332.2014.942901

Mailey, E. L., \& Hsu, W. W. (2017). Is a general or specific exercise recommendation more effective for promoting physical activity among postpartum mothers? Journal of Health Psychology, 1-15. https://doi. org/10.1177/1359105316687627

Mailey, E. L., Huberty, J., Dinkel, D., \& McAuley, E. (2014). Physical activity barriers and facilitators among working mothers and fathers. BMC Public Health, 1-9. https://doi.org/10.1186/1471-2458-14-657

Mailey, E. L., Huberty, J., \& Irwin, B. C. (2016). Feasibility and effectiveness of a web-based physical activity intervention for working mothers. Journal of Physical Activity and Health, 13(8), 822-829. https:// doi.org/10.1123/jpah.2015-0643

Mailey, E. L., \& McAuley, E. (2014). Impact of a brief intervention on physical activity and social cognitive determinants among working mothers: A randomized trial. Journal of Behavioral Medicine, 37(2), 343-355. https://doi.org/10.1007/s10865-013-9492-y

Mansfield, E. D., Ducharme, N., \& Koski, K. G. (2012). Individual, social and environmental factors influencing physical activity levels and behaviours of multiethnic socio-economically disadvantaged urban mothers in Canada: A mixed methods approach. International Journal of Behavioral Nutrition and Physical Activity, 9, 1-15. https://doi.org/10.1186/1479-5868-9-42

Mascarenhas, M. N., Chan, J. M., Vittinghoff, E., Van Blarigan, E. L., \& Hecht, F. (2018). Increasing physical activity in mothers using video exercise groups and exercise mobile apps: Randomized controlled trial. Journal of Medical Internet Research, 20(5), 1-14. https://doi.org/10.2196/jmir.9310

McGannon, K. R., \& Schinke, R. J. (2013). “ My first choice is to work out at work; then i don't feel bad 
about my kids": A discursive psychological analysis of motherhood and physical activity participation. Psychology of Sport and Exercise, 14(2), 179-188. https://doi.org/10.1016/j.psychsport.2012.10.001

Mcquillan, J., Greil, A. L., Scheffler, K. M., \& Tichenor, V. (2010). The Importance of Motherhood among Women in the Contemporary United States. Gender \& Society, 22(4), 1-17.https://doi.org/10.1177/0891243208319359.

Miller, Y. D., Trost, S. G., \& Brown, W. J. (2002). Mediators of physical activity behavior change among women with young children. American Journal of Preventive Medicine, 23(2, Suppl. 1), 98-103. https:// doi.org/10.1016/S0749-3797(02)00484-1

Moher, D., Liberati, A., Tetzlaff, J., Altman, D. G., \& Prisma Group. (2009). Preferred reporting items for systematic reviews and meta-analyses: The PRISMA Statement (Reprinted from Annals of Internal Medicine). Annals of Internal Medicine, 151(4), 264-269. https:// doi.org/10.1371/journal.pmed.1000097

Nomaguchi, K. M., \& Bianchi, S. M. (2004). Exercise time: Gender differences in the effects of marriage, parenthood, and employment. Journal of Marriage and Family, 66(2), 413-430. https://doi.org/10.1111/j.17413737.2004.00029.x

Norman, E., Sherburn, M., Osborne, R. H., \& Galea, M. P. (2010). An exercise and education program improves well-being of new mothers: A randomized controlled trial. Physical Therapy, 90(3), 348-355. https://doi. org/10.2522/ptj.20090139

Olvera, N., Smith, D. W., Lee, C., Liu, J., Lee, J., Kim, J. H., \& Kellam, S. F. (2011). Comparing high and low acculturated mothers and physical activity in Hispanic children. Journal of Physical Activity \& Health, 8(Suppl. 2), S206-S213.

Ransdell, L. B., Taylor, A., Oakland, D., Schmidt, J., Moyer-Mileur, L., \& Shultz, B. (2003). Daughters and mothers exercising together: Effects of home- and community-based programs. Medicine and Science in Sports and Exercise, 35(2), 286-296. https://doi. org/10.1249/01.MSS.0000048836.67270.1F

Rhodes, R. E., Blanchard, C. M., Benoit, C., LevyMilne, R., Naylor, P. J., Symons Downs, D., \& Warburton, D. E. R. (2014). Belief-level markers of physical activity among young adult couples: comparisons across couples without children and new parents. Psychology \& Health, 29(11), 1320-1340. https://doi.org/10.1080/08870446.2014.929687
Saligheh, M., McNamara, B., \& Rooney, R. (2016). Perceived barriers and enablers of physical activity in postpartum women: A qualitative approach. $B M C$ Pregnancy and Childbirth, 16(1), 1-8. https://doi. org/10.1186/s12884-016-0908-x

Sallis, J. F., Greenlee, L., McKenzie, T. L., Broyles, S. L., Zive, M. M., Berry, C. C., Brennan, J., \& Nader, P. R. (2001). Changes and Tracking of physical activity across seven years in Mexican-American and European-American mothers. Women \& Health, 34(4), 1-14. https://doi.org/10.1300/J013v34n04

Schluter, P., Oliver, M., \& Paterson, J. (2011). Perceived barriers and incentives to increased physical activity for Pacific mothers in New Zealand: Findings from the Pacific Islands families study. Australian and New Zealand Journal of Public Health, 35(2), 151-158. https://doi.org/10.1111/j.1753-6405.2011.00685.x

Suzuki, Y., Sakuraba, K., Shinjo, T., MaruyamaNagao, A., Nakaniida, A., Kadoya, H., Shibata, M., Matsukawa, T., Itoh, H., \& Yokoyama, K. (2017). Physical inactivity associated with the risk of non-communicable diseases in Japanese working mothers with young children: A cross-sectional study in Nagano city, Japan. Experimental and Therapeutic Medicine, 13(6), 31033108. https://doi.org/10.3892/etm.2017.4311

Tuominen, P. P. A., Husu, P., Raitanen, J., Kujala, U. M., \& Luoto, R. M. (2017). The effect of a movement-to-music video program on the objectively measured sedentary time and physical activity of preschool-aged children and their mothers: A randomized controlled trial. PLoS ONE, 12(8), 1-21. https://doi.org/10.1371/journal.pone.0183317

Tuominen, P. P. A., Husu, P., Raitanen, J., \& Luoto, R. M. (2016). Differences in sedentary time and physical activity among mothers and children using a movementto-music video program in the home environment: A pilot study. SpringerPlus, 5(1), 1-10. https://doi.org/10.1186/ s40064-016-1701-z

U.S. Department of Health and Human Services. (2018). Physical Activity Guidelines for Americans (2nd ed.). U.S. Department of Health and Human Services. https:// doi.org/10.1249/fit.0000000000000472

World Health Organization (WHO). (2011). Global Recommendations on Physical Activity for Health: 18-64 years old. https://doi.org/10.1080/11026480410034349

World Health Organization (WHO). (2018). Global Action Plan on Physical Activity 2018-2030: More Active People for A Healthier World. https://doi.org/10.1016/j. jpolmod.2006.06.007 